\title{
Códigos de peso constante
}

\author{
Ruth Nascimento
}

\section{TESE APRESENTADA}

$\mathrm{AO}$

Instituto de MATemática e Estatística

DA

Universidade de SÃo Paulo

PARA

OBTENÇÃO DO TÍTULO

$\mathrm{DE}$

Doutor EM Cî̂nCIAS

\author{
Programa: Matemática \\ Orientador: Prof. Dr. Raul Antonio Ferraz
}

Durante o desenvolvimento deste trabalho a autora recebeu auxílio financeiro da CAPES e do CNPQ

São Paulo, agosto de 2014 


\section{Códigos de peso constante}

Esta versão definitiva da tese contém as correções e alterações sugeridas pela Comissão Julgadora durante a defesa realizada por Ruth Nascimento em 09/06/2014.

Comissão Julgadora:

- Prof. Dr. Raul Antonio Ferraz (orientador) - IME-USP.

- Prof. Dr. Francisco César Polcino Milies - IME-USP.

- Profa. Dra. Sueli Irene Rodrigues Costa- UNICAMP.

- Profa. Dra. Marinês Guerreiro - UFV.

- Prof. Dr. Thierry Corrêa Petit Lobão - UFBA. 


\section{Resumo}

Sejam $\mathcal{F}_{q}$ um corpo finito com $q$ elementos, e $C_{n}$ um grupo cíclico de $n$ elementos com $m d c(q, n)=1$, Iniciamos nosso trabalho inspirados nos resultados de Vega [19], estabelecendo condições para que um código de $\mathcal{F}_{q} C_{n}$ tenha peso constante. Com tal resultado concluímos que um código de peso constante em $\mathcal{F}_{q} C_{n}$ é da forma $\left\{r g^{i} e \mid r \in\right.$ $\left.\mathcal{F}_{q}, 0 \leq i \leq n\right\}$. A partir disto, determinamos a quantidade de códigos de peso constante de $\mathcal{F}_{q} C_{n}$, e construímos exemplos de códigos de dois pesos em $\mathcal{F}_{q}\left(C_{n} \times C_{n}\right)$. Em seguida, estabelecemos sob quais condições um código em $\mathcal{F}_{q} A$, para $A$ um grupo abeliano finito, tem peso constante. Analisamos também os códigos de peso constante em $R G$, quando $R$ um anel de cadeia finito e $C_{n}$ é um grupo cíclico de $n$ elementos com $m d c(n, q)=1$. Além disso, analisamos o caso em que os elementos de um ideal de $R A$, para $R$ um domínio de integridade infinito e $A$ um grupo abeliano finito têm peso constante.

Palavras-chave: Códigos de peso constante, anéis de grupo, anéis de cadeia, grupo cíclico, grupo abeliano. 


\section{Abstract}

Let $\mathcal{F}_{q}$ be a field with $q$ elements, $C_{n}$ be a cyclic group of order $n$ and suppose that $\operatorname{gcd}(q, n)=1$. In this work conditions are given to ensure that a code in $\mathcal{F}_{q} C_{n}$ is a one weight code, inspired in the work of Vega [19]. As a consequence of this result we showed that a one weight code in $\mathcal{F}_{q} C_{n}$ is of the form $\left\{r g^{i} e \mid r \in \mathcal{F}_{q}, 0 \leq i \leq n\right\}$. With this, we determined the number of one weight codes in $\mathcal{F}_{q} C_{n}$, and constructed examples of two weight codes in $\mathcal{F}_{q}\left(C_{n} \times C_{n}\right)$. After this, we gave conditions to ensure that a code had constant weight in $\mathcal{F}_{q} A$, for $A$ a finite abelian group. We also analyzed the one weight codes in $R G, R$ a chain ring and $C_{n}$ a cyclic group with $n$ elements with $\operatorname{gcd}(n, q)=1$. Moreover, we analyzed the case when the elements of an ideal in $R A$, for $R$ an infinite integral domain and $A$ a finite abelian group, have constant weight.

Key words: One weight codes, group rings, chain ring, cyclic group, abelian group. 


\section{Sumário}

Introdução $\quad$ x

1 Preliminares 1

1.1 Grupos . . . . . . . . . . . . . . . . . . 1

1.2 Anéis e Anéis de grupo . . . . . . . . . . . . . . . . 3

1.3 Códigos . . . . . . . . . . . . . . . . . . . . . . . . 10

2 Códigos Cíclicos de Peso Constante 15

2.1 Quando o código tem peso constante . . . . . . . . . . . 15

2.2 Quantidade de códigos de peso constante . . . . . . . . . . . . . 21

2.3 Códigos de dois pesos . . . . . . . . . . . . . . . . . 22

3 Códigos de peso constante em outras classes de anéis de grupos 28

3.1 Grupo Abeliano . . . . . . . . . . . . . . . . . . . . . . . . . . 28

3.2 Domínio de Integridade . . . . . . . . . . . . . . . . . 31

3.3 Anel de Cadeia . . . . . . . . . . . . . . . . . . . . . . . . . . . . 33

$\begin{array}{llr}4 & \text { Conclusão } & 39\end{array}$

$\begin{array}{ll}\text { Referências Bibliográficas } & 40\end{array}$ 


\section{Introdução}

Ao se tentar transmitir uma informação, que consiste de uma sequência finita de símbolos que são elementos de um alfabeto finito, podem ocorrer erros de tal modo que o receptor da mensagem receba uma informação distorcida, podendo assim cometer erros na interpretação da informação. A Teoria de Códigos auxilia para que estes erros tornem-se menos frequentes, ajudando a detectar e corrigir os erros ocorridos na transmissão da informação.

Segundo [13], um dos marcos iniciais desta teoria é um famoso teorema de C.E. Shannon, encontrado no artigo intitulado "A Mathematical Theory of Communication", também publicado no "The Bell System Technical Journal"[16], que garante a existência de códigos que podem transmitir informação com uma probabilidade arbitrariamente pequena de erro, sendo um campo de pesquisa muito ativo na atualidade em diversas áreas do conhecimento, como Matemática, Computação, Engenharia Elétrica e Estatística. Um dos propósitos da Teoria Algébrica de Códigos é desenvolver métodos para a construção de tais códigos.

É importante na teoria o estudo do peso de Hamming de um código. Em particular, temos os códigos de peso constante, isto é, aqueles em que todas as suas palavras não nulas têm o mesmo peso de Hamming. Muitos dos trabalhos nesta área estão baseados no estudo de códigos binários de peso constante, que possui várias aplicações, como por exemplo na comunicação móvel. Atualmente, tem crescido também o interesse em códigos não binários de peso constante, pelo crescente uso de alfabetos não binários 
(veja por exemplo [3]). Mas tais códigos foram bem menos explorados comparados com os códigos binários.

Em nosso trabalho estudamos códigos, não necessariamente binários, em determinados anéis de grupo, buscando inicialmente dar condições para que tais códigos tenham peso constante.

O primeiro capítulo traz os resultados e definições encontrados na bibliografia e que serão utilizados ao longo do texto.

No segundo capítulo, analisamos os códigos de peso constante em $\mathcal{F}_{q} C_{n}$, para $\mathcal{F}_{q}$ um corpo com $q$ elementos e $C_{n}$ um grupo cíclico de $n$ elementos com $m d c(q, n)=1$, buscando dar condições para garantir que um código de $\mathcal{F}_{q} C_{n}$ tenha peso constante. Tal trabalho foi baseado no seguinte resultado de Vega [19]:

Teorema 0.1 ([19], Teorema 9). Seja $\mathcal{F}_{q}$ um corpo com $q$ elementos, $n=\lambda \frac{q^{k}-1}{q-1}$, com $\lambda$ dividindo $(q-1)$, e $\mathcal{C}$ um código cíclico de $\mathcal{F}_{q}$ de comprimento $n$ e dimensão $k$, gerado por $g(x)$, e com polinômio de checagem $h(x)$. Então, $\mathcal{C}$ tem peso constante se, e somente se, $w(g e)=\lambda\left(q^{k-1}\right)$.

Nosso trabalho deu origem ao seguinte resultado:

Teorema 0.2. Sejam $\mathcal{F}_{q}$ um corpo com $q$ elementos, $n$ um inteiro positivo tal que $\operatorname{mdc}(q, n)=1, C_{n}=<g>$ um grupo cíclico com $n$ elementos, e $\mathcal{F}_{q} C_{n}$ o anel de grupo de $C_{n}$ sobre $\mathcal{F}_{q}$. Considere e um idempotente primitivo de $\mathcal{F}_{q} C_{n}, \mathcal{C}=\mathcal{F}_{q} C_{n}$ e o respectivo código irredutível e $\operatorname{dim}_{\mathcal{F}_{q}} \mathcal{C}=k$. São equivalentes:

1. $\mathcal{C}$ tem peso constante;

2. Todo elemento não nulo de $\mathcal{C}$ tem peso $\frac{q^{k-1}(q-1) n}{q^{k}-1}$;

3. Existe um elemento de $\mathcal{C}$ cujo peso é $\frac{q^{k-1}(q-1) n}{q^{k}-1}$;

4. $\left(\mathcal{F}_{q} C_{n} e\right)^{*}=\mathcal{F}_{q}^{*} e C_{n} e$.

Como consequência de tal resultado concluímos que os códigos de peso constante de $\mathcal{F}_{q} C_{n}$, com $m d c(n, q)=1$, são da forma $\left\{r g^{i} e \mid r \in \mathcal{F}_{q}, 0 \leq i \leq n\right\}$. Em seguida, 
chegamos a uma fórmula que determina a quantidade de códigos de peso constante em $\mathcal{F}_{q} C_{n}$. Finalizando o capítulo, construímos exemplos de códigos de dois pesos em $\mathcal{F}_{q}\left(C_{n} \times C_{n}\right)$.

O terceiro capítulo foi destinado a dar condições que garantissem que um código é de peso constante em outras classes de anéis de grupo. Analisamos os códigos de peso constante nos anéis de grupo $\mathcal{F}_{q} A$, com $A$ um grupo abeliano finito, $R G$, com $R$ um anel de cadeia finito e $G$ um grupo cíclico de ordem $n, \operatorname{com} m d c(n, q)=1$, além de analisar os ideais de peso constante de $R G$, para $R$ um domínio de integridade infinito e $G$ um grupo abeliano finito. 


\section{Capítulo 1}

\section{Preliminares}

Daremos aqui as principais definições e resultados encontrados na bibliografia [8], [9], [10], [14], [15] e [17], e que serão usados ao longo do texto.

\subsection{Grupos}

Definição 1.1. Um grupo $(G, \cdot)$ consiste de um conjunto $G$, fechado em relação à operação binária $\cdot$, tal que os seguintes axiomas são satisfeitos:

1. A operação binária $\cdot$ é associativa.

2. Existe um elemento $1 \in G$ tal que $1 \cdot x=x \cdot 1=x$, para todo $x \in G$ (tal elemento é chamado elemento identidade de $(G, \cdot))$.

3. Para cada a $\in G$, existe um elemento $a^{\prime} \in G$ com a propriedade que $a \cdot a^{\prime}=a^{\prime} \cdot a=1$ (tal elemento $a^{\prime}$ é chamado inverso de a com respeito à operação ·).

Denotaremos o grupo $(G, \cdot)$ por $G$.

Se a operação · é comutativa o grupo $G$ é dito abeliano.

Definição 1.2. Dados $G, G^{\prime}$ dois grupos, uma função $f: G \rightarrow G^{\prime}$ é um homomorfismo de grupos se, dados $a, b \in G, f(a b)=f(a) f(b)$. Se $f$ é também bijetora 
então temos um isomorfismo de grupos, e dizemos que $G$ é isomorfo a $G^{\prime}$, o que denotamos por $G \simeq G^{\prime}$.

A ordem de um grupo $G$ é a quantidade de elementos de $G$, que pode ser finita ou infinita. Neste trabalho, todos os grupos considerados têm ordem finita. Denotamos por $|G|$ a ordem do grupo $G$.

Dado $g \in G$, caso exista um natural $n$ tal que $g^{n}=1$, o menor natural positivo tal que isso ocorre é chamado de ordem de $g$.

Definição 1.3. Um elemento a de um grupo $G$ gera $G$ se $\langle a\rangle=\left\{a^{n} \mid n \in \mathbb{Z}\right\}=G$. Um grupo $G$ é dito cíclico se existe algum $a \in G$ que gera $G$.

Definição 1.4. Seja $H$ um subgrupo de um grupo $G$ e a um elemento de $G$. O subconjunto $a H=\{a h \mid h \in H\}$ é a classe lateral à esquerda de $H$ contendo a enquanto $H a=\{h a \mid h \in H\}$ é $a$ classe lateral à direita de $H$ contendo a.

Se $G$ é um grupo abeliano $a H=H a$ é simplesmente a classe lateral de $H$ contendo $a$.

Definição 1.5. Um subgrupo $H$ de um grupo $G$ é normal se, para todo a $\in G$, $a H=H a$, ou equivalentemente, se $a H a^{-1} \subseteq H$, para todo $a \in G$.

Note que se $G$ é abeliano, todo subgrupo de $G$ é normal em $G$.

Definição 1.6. Seja $H$ subgrupo normal de um grupo $G$. Definimos um novo grupo $G / H$, cujos elementos são as classes aH, $a \in G$, e a operação é dada por

$$
(a H)(b H)=(a b H), \text { para todos } a, b \in G .
$$

Notação: Denotaremos por $[G: H]$ o índice de $H$ em $G$, que é o número de classes laterais de $H$ em $G$.

Teorema 1.7. ([8], Teorema 2.5)[Teorema de Lagrange] Seja H um subgrupo de um grupo finito $G$. Então, a ordem de $H$ divide a ordem de $G$. Mais precisamente, temos

$$
|G|=[G: H]|H|
$$

Teorema 1.8. Seja $H$ um subgrupo normal de um grupo G. Então 
1. Para cada subgrupo $K$ de $G$ contendo $H$, o conjunto $\frac{K}{H}=\{x H: x \in K\}$ é um subgrupo de $\frac{G}{H}$.

2. Reciprocamente, se $\mathcal{K}$ é um subgrupo do grupo quociente $\frac{G}{H}$, então a pré-imagem $K=\{x \in G: x H \in \mathcal{K}\}$ é um subgrupo de $G$ contendo $H$ tal que $\mathcal{K}=\frac{K}{H}$.

Definição 1.9. Dado um grupo $G$, definimos o expoente de $G$ como o menor inteiro positivo $m$ tal que $g^{m}=1$, para todo $g \in G$, caso tal número exista.

Teorema 1.10. Se $H_{1}, H_{2}<G, G$ cíclico finito, então $\left|H_{1} H_{2}\right|=m m c\left(\left|H_{1}\right|,\left|H_{2}\right|\right)$, e $\left|H_{1} \cap H_{2}\right|=m d c\left(\left|H_{1}\right|,\left|H_{2}\right|\right)$.

Prova: Pelo Teorema de Lagrange, $\left|H_{1}\right||| H_{1} H_{2} \mid$ e $\left|H_{2}\right||| H_{1} H_{2} \mid$, donde $m m c\left(\left|H_{1}\right|,\left|H_{2}\right|\right)|| H_{1} H_{2} \mid$. Como $H_{1} H_{2}$ é subgrupo de $G$, então $H_{1} H_{2}$ é cíclico já que $G$ é cíclico. Digamos $H_{1} H_{2}=<\alpha>$, com $\alpha=\beta_{1} \beta_{2}, \beta_{1} \in H_{1}, \beta_{2} \in H_{2}$. Logo

$$
\alpha^{m m c\left(\left|H_{1}\right|\left|H_{2}\right|\right)}=\left(\beta_{1} \beta_{2}\right)^{m m c\left(\left|H_{1}\right|\left|H_{2}\right|\right)}=\beta_{1}^{m m c\left(\left|H_{1}\right|\left|H_{2}\right|\right)} \beta_{2}^{m m c\left(\left|H_{1}\right|\left|H_{2}\right|\right)}=1,
$$

donde $\left|H_{1} H_{2}\right|=o(\alpha) \mid m m c\left(\left|H_{1}\right|\left|H_{2}\right|\right)$, e portanto $\left|H_{1} H_{2}\right|=m m c\left(\left|H_{1}\right|\left|H_{2}\right|\right)$. Como $\left|H_{1}\right|\left|H_{2}\right|=m m c\left(\left|H_{1}\right|,\left|H_{2}\right|\right) m d c\left(\left|H_{1}\right|,\left|H_{2}\right|\right)$, bem como $\left|H_{1}\right|\left|H_{2}\right|=\left|H_{1} H_{2}\right|\left|H_{1} \cap H_{2}\right|$ o resultado segue.

\subsection{Anéis e Anéis de grupo}

Definição 1.11. Um anel $(R,+, \cdot)$ consiste de um conjunto não vazio $R$ munido de duas operações binárias $+e \cdot$, que chamamos adição $e$ multiplicação, tal que os seguintes axiomas são satisfeitos:

1. $(R,+)$ é um grupo abeliano.

2. a multiplicação é associativa.

3. Para todo $a, b, c \in R$, a lei distributiva à esquerda, $a \cdot(b+c)=a \cdot b+a \cdot c, e$ $a$ lei distributiva à direita, $(a+b) \cdot c=a \cdot c+b \cdot c$, ocorrem. 
Denotaremos $(R,+, \cdot)$ por $R$. Se existe um elemento $1 \in R$ tal que $1 \cdot x=x \cdot 1=x$, para todo $x \in R$, então $R$ é dito um anel com unidade e, se a operação · é comutativa, $R$ é dito anel comutativo. Ao longo do texto, por anel estaremos entendendo anel comutativo com unidade. Um corpo é um anel comutativo com unidade tal que todo elemento não nulo possui um inverso multiplicativo.

Definição 1.12. Dados $R, R^{\prime}$ anéis, uma função $f: R \rightarrow R^{\prime}$ é um homomorfismo de anéis se, para todos $a, b \in R, f(a+b)=f(a)+f(b)$ e $f(a b)=f(a) f(b)$. Se $f$ é também bijetora, então $f$ é um isomorfismo de anéis. A notação $R \simeq R^{\prime}$ significa que existe um isomorfismo entre os anéis $R$ e $R^{\prime}$ que são, assim, ditos isomorfos.

Teorema 1.13. Se $K$ é um corpo finito, o conjunto $K^{*}=K-\{0\}$ é um grupo cíclico com a operação multiplicação de $K$.

Definição 1.14. Dado um anel comutativo com unidade $R$, um conjunto $M$ é dito um R-módulo se $M$ é um grupo abeliano e se existe uma função $\cdot: R \times M \rightarrow M$ satisfazendo, para todos $x, y \in R, m, n \in M$ :

1. $(x+y) m=x m+y m$.

2. $x(m+n)=x m+x n$.

3. $(x y) m=x(y m)$.

4. $1 m=m$.

Definição 1.15. Dados dois R-módulos $M, M^{\prime}$, uma função $f: M \rightarrow M^{\prime}$ é um homomorfismo de $R$-módulos se é um homomorfismo de grupos aditivos que satisfaz $f(a m)=a f(m)$, para todos $a \in R, m \in M$. Se $f$ é bijetora é chamada de isomorfismo de R-módulos.

Definição 1.16. Um ideal de um anel $R$ (comutativo) é um subconjunto $I$ de $R$, fechado para a soma de $R$ e tal que, para todo $r \in R, x \in I, r x \in I$.

Definição 1.17. Dados $I_{1}, I_{2}$ ideais de um anel $R$, o ideal $I_{1}+I_{2}$ é denotado por $I_{1} \oplus I_{2}$ e dito soma direta de $I_{1}$ e $I_{2}$, se $I_{1} \cap I_{2}=\{0\}$.

Definição 1.18. Um ideal é dito indecomponível se é diferente de 0 e não pode ser escrito como soma direta de ideais não nulos. 
Definição 1.19. Um domínio de integridade $D$ é um anel comutativo com unidade sem divisores de zero, isto é, sem elementos não nulos $a, b$ tais que $a b=0$.

Definição 1.20. A característica de um anel $R$ é o menor inteiro positivo tal que $n r=0$, para todo $r \in R$. Se um tal inteiro não existe, dizemos que $R$ tem característica 0 .

Denotaremos a característica de um anel por $\operatorname{char}(R)$.

Teorema 1.21. A característica de um domínio de integridade é 0 ou um número primo.

Definição 1.22. Um elemento e de um anel $R$ é chamado idempotente se $e^{2}=e$. Dois idempotentes $e_{1}$ e $e_{2}$ são chamados ortogonais se $e_{1} \cdot e_{2}=0$. Um idempotente e é chamado primitivo se não existem $e_{1}, e_{2}$ idempotentes ortogonais não nulos tais que $e=e_{1}+e_{2}$. Um conjunto de idempotentes de um anel com unidade $\left\{e_{1}, \cdots, e_{n}\right\}$ é um conjunto completo de idempotentes primitivos ortogonais se $e_{1}+\cdots+e_{n}=1$, cada $e_{i}$ é primitivo e $e_{i} e_{j}=0$, se $i \neq j$, para $1 \leq i, j \leq n$.

Proposição 1.23. Se $R=I_{0} \oplus \cdots \oplus I_{j}$, $I_{k}$ ideais de $R, 1 \leq k \leq j$, então existem idempotentes ortogonais $e_{0}, \cdots, e_{j}$ tais que $1=e_{0}+\cdots+e_{j}$ e $e_{k} R=I_{k}$, para $1 \leq k \leq j$.

Definição 1.24. Um elemento a em um anel $R$ é dito nilpotente se existe $n \in \mathbb{N}$ tal que $a^{n}=0$. O menor inteiro positivo $m$ tal que $a^{m}=0$ é chamado índice de nilpotência de a em $R$.

Definição 1.25. Seja $R$ um anel. O radical de Jacobson de $R$, denotado por $J(R)$, é a interseção de todos os ideais maximais de $R$.

Definição 1.26. Um anel $R$ é dito local se possui um único ideal maximal.

Teorema 1.27 (Lema de Nakayama). Seja $R$ um anel e $M$ um $R$-módulo finito e $I$ um ideal de $R$. Suponha que $I M=M$. Então existe um elemento a $\in R$ da forma $a=1+x, x \in I$, tal que $a M=0$. Se além disso $I \subset \operatorname{rad}(R)$, então $M=0$.

Teorema 1.28. ([9], Proposição 7.14) Seja $R$ anel comutativo e $N$ um nil ideal em $R$, isto é, um ideal cujos seus elementos são todos nilpotentes, e seja $f=u+N$ um idempotente de $\bar{R}=\frac{R}{N}$. Então existe um único idempotente e em $R$ tal que $f=\bar{e}$. 
Definição 1.29. Um anel comutativo $R$ é dito simples se $R$ é não nulo e não possui ideais além de $(0)$ e ele próprio.

Definição 1.30. Um anel $R$ (comutativo) é dito semisimples se qualquer de seus ideais é um somando direto, isto é, se para qualquer ideal $I$ de $R$, existe um ideal $J$ de $R$ tal que $R=I \oplus J$.

Definição 1.31. Um anel comutativo $R$ é chamado anel de cadeia se o conjunto de todos os seus ideais forma uma cadeia com a relação de inclusão.

Exemplo 1.32. $R=\frac{\mathbb{Z}_{p}[x]}{\left\langle x^{r}\right\rangle}$ é um anel de cadeia, com a cadeia de ideais

$$
R=\langle 1\rangle \supseteq\langle x\rangle \supseteq \cdots \supseteq\left\langle x^{r}\right\rangle=0 .
$$

Teorema 1.33. ([4] Proposição 2.1) Para um anel finito $R$ as seguintes condições são equivalentes:

1. $R$ é um anel local e o ideal maximal $M$ de $R$ é principal, isto é, tem um conjunto gerador unitário.

2. $R$ é um anel local de ideais principais.

3. $R$ é um anel de cadeia.

Dado um conjunto finito $C$, denotamos por $|C|$ a cardinalidade de $C$, isto é, a quantidade de elementos de $C$.

Temos o seguinte:

Teorema 1.34. ([4], Proposição 2.2) Seja $R$ um anel de cadeia finito e comutativo com unidade, com ideal maximal $M=\langle a\rangle, t$ indice de nilpotência de a em $R$ e $\bar{R}=\frac{R}{M}$. Então:

1. Para algum primo $q$ e inteiros positivos $k$ e $l,(k \geq l),|R|=q^{k},|\bar{R}|=q^{l}$ e a característica de $R$ e $\bar{R}$ são potências de $q$.

2. Para $i=0,1, \cdots, t,\left|a^{i}\right|=|\bar{R}|^{t-i}$. Em particular, $|R|=|\bar{R}|^{t}$, isto é, $k=l t$.

Dado $R$ um anel e $G$ um grupo, podemos definir um novo conjunto, que denotamos por $R G$, cujos elementos são da forma $\sum_{g \in G} \alpha_{g} g$, com $\alpha_{g} \in R$. 
Definição 1.35. Dado um elemento $\alpha=\sum_{g \in G} a_{g} g$ de $R G$, o suporte de $\alpha$, denotado por $\operatorname{supp}(\alpha)$ é dado por

$$
\operatorname{supp}(\alpha)=\left\{g \in G: a_{g} \neq 0\right\}
$$

Definição 1.36. Dado $G$ um grupo e $R$ um anel, definimos um novo anel $R G$ cujos elementos são combinações formais de suporte finito

$$
\alpha=\sum_{g \in G} a_{g} g
$$

com soma dada por

$$
\sum_{g \in G} a_{g} g+\sum_{g \in G} b_{g} g=\sum_{g \in G}\left(a_{g}+b_{g}\right) g
$$

e produto dado por

$$
\left(\sum_{g \in G} a_{g} g\right)\left(\sum_{h \in G} b_{h} h\right)=\sum_{g, h \in G} a_{g} b_{h} g h .
$$

Um tal anel é chamado anel de grupo.

Dados dois elementos $\alpha=\sum_{g \in G} a_{g} g$ e $\beta=\sum_{g \in G} b_{g} g$ temos $\alpha=\beta$ se, e somente se, $a_{g}=b_{g}$, para todo $g \in G$.

Definição 1.37. O homomorfismo

$$
\begin{aligned}
\epsilon: R G & \rightarrow R \\
\left(\sum_{g \in G} a_{g} g\right) & \mapsto \sum_{g \in G} a_{g}
\end{aligned}
$$

é chamado aplicação de aumento e seu núcleo, dado por $\operatorname{Ker}(\epsilon)=\left\{\sum_{g \in G} a_{g}(g-1)\right.$ : $g \in G, g \neq 1\}$, é chamado ideal de aumento de $R G$.

Um dos resultados básicos da teoria de anéis de grupo é o seguinte:

Teorema 1.38. ([14] Teorema 3.4.7)(Teorema de Maschke) Seja G um grupo. Então, o anel de grupo $R G$ é semisimples se, e somente se, as seguintes condições ocorrem:

1. $R$ é um anel semisimples.

2. G é finito.

3. $|G|$ é invertível em $R$. 
Um caso de particular importância é o seguinte:

Corolário. Seja $G$ um grupo finito e $K$ um corpo. Então $K G$ é semisimples se, e somente se, char $(K)$ não divide $|G|$.

Corolário. Se $G$ é um grupo abeliano e $K$ é um corpo tal que car $(K)$ não divide $|G|$, então KG é isomorfo a uma soma direta de corpos.

Vale o seguinte

Teorema 1.39. ([17], Teorema 2.1.3) Seja $R$ um anel de cadeia finito, comutativo e com unidade, com $|R|=q^{k}, M=\langle a\rangle$ ideal maximal de $R$ e t o indice de nilpotência de a em $R$. Seja $G=C_{n}$, em que $q$ não divide $n$. Se I é um ideal de $R G e_{i}, e_{i}$ idempotente primitivo, então $I$ é da forma $I=\left\langle a^{k_{j}} e_{i}\right\rangle$, com $0 \leq k_{j} \leq t$.

Proposição 1.40. Se I é um ideal bilateral de um anel $R$ e $G$ é um grupo comutativo, então $I G=\left\{\sum_{g \in G} a_{g} g: a_{g} \in G\right\}$ é um ideal bilateral de $R G$ e $\frac{R G}{I G} \simeq\left(\frac{R}{I}\right) G$.

Teorema 1.41. ([11], Teorema VII.8) Seja e um idempotente central não nulo de um anel $R G$. As seguintes condições são equivalentes:

1. e é primitivo.

2. RGe é um anel local.

3. RGe é indecomponivel.

Para um anel de grupo semisimples, sempre existe $\left\{e_{1}, e_{2}, \ldots, e_{m}\right\}$ um conjunto completo de idempotentes primitivos ortogonais de $R G$ tal que

$$
R G=R G e_{1} \oplus R G e_{2} \oplus \cdots \oplus R G e_{m}
$$

Para anéis locais, em particular para anéis de cadeia, vale o seguinte.

Teorema 1.42. ([17], Teorema 2.1.2) Sejam $R$ um anel local com ideal maximal $M=\langle a\rangle$, com $|R|=q^{k}$ e $G$ um grupo cíclico de ordem $n$, tal que $q$ não divide $n$. Se $\left\{\overline{e_{0}}, \cdots, \overline{e_{m}}\right\}$ é um conjunto de idempotentes primitivos ortogonais de $\bar{R} G$, então $\left\{e_{0}, \cdots, e_{m}\right\}$ é um conjunto de idempotentes primitivos ortogonais de $R G$. 
Um último resultado estrutural sobre anéis de grupo é o seguinte.

Teorema 1.43. ([18] Proposição 1.3.1) Seja $R$ um anel comutativo com unidade, $G$ e $H$ grupos. Então $R(G \times H) \simeq(R G) H$.

Prova: Considere a função:

$$
\begin{array}{r}
\varphi: R(G \times H) \rightarrow(R G) H \\
\sum_{g \in G, h \in H} \alpha_{(g, h)}(g, h) \mapsto \sum_{h \in H}\left(\sum_{g \in G} \alpha_{(g, h)} g\right) h
\end{array}
$$

Temos que $\varphi$ está bem definida. Vejamos que é um homomorfismo de anéis. Sejam $\alpha=\sum_{g \in G, h \in H} \alpha_{(g, h)}(g, h)$ e $\beta=\sum_{g \in G, h \in H} \beta_{(g, h)}(g, h) \in R(G \times H)$, Então $\alpha+\beta=$ $\sum_{g \in G, h \in H}\left(\alpha_{(g, h)}+\beta_{(g, h)}\right)(g, h)$ bem como $\alpha \beta=\sum_{g_{1}, g_{2} \in G, h_{1}, h_{2} \in H} \alpha_{\left(g_{1}, h_{1}\right) \beta_{\left(g_{2}, h_{2}\right)}}\left(g_{1} g_{2}, h_{1} h_{2}\right)$. Logo,

$$
\begin{gathered}
\varphi(\alpha+\beta)=\sum_{h \in H}\left(\sum_{g \in G}\left(\alpha_{(g, h)}+\beta_{(g, h)}\right) g\right) h=\sum_{h \in H}\left(\sum_{g \in G} \alpha_{(g, h)} g\right) h+\sum_{h \in H}\left(\sum_{g \in G} \alpha_{(g, h)} g\right) h \\
=\varphi(\alpha)+\varphi(\beta) .
\end{gathered}
$$

Também

$$
\varphi(\alpha \beta)=\sum_{h_{1}, h_{2} \in H}\left(\sum_{g \in G} \alpha_{\left(g_{1}, h_{1}\right)} \beta_{\left(g_{2}, h_{2}\right)} g_{1} g_{2}\right) h_{1} h_{2} .
$$

Por outro lado,

$\varphi(\alpha) \varphi(\beta)=\left[\sum_{h \in H}\left(\sum_{g \in G} \alpha_{(g, h)} g\right) h\right]\left[\sum_{h \in H}\left(\sum_{g \in G} \beta_{(g, h)} g\right) h\right]=\sum_{h_{1}, h_{2} \in H}\left(\sum_{g \in G} \alpha_{\left(g_{1}, h_{1}\right)} \beta_{\left(g_{2}, h_{2}\right)} g_{1} g_{2}\right) h_{1} h_{2}$.

Donde $\varphi(\alpha \beta)=\varphi(\alpha) \varphi(\beta)$. Além disso, $\varphi$ é injetora, pois se $\alpha \sum_{g \in G, h \in H} \alpha_{(g, h)}(g, h) \in$ $R(G \times H)$ é tal que $\varphi(\alpha)=\sum_{h \in H}\left(\sum_{g \in G} \alpha_{(g, h)} g\right) h=0$, como $H$ gera $(R G) H$ sobre $R G$ então $\sum_{g \in G} \alpha_{(g, h)} g=0$, para todo $h \in H$ e, portanto, $\alpha_{(g, h)}=0$, para todos $g \in G, h \in H$ e, assim, $\alpha=0$. Por fim, $\varphi$ é sobrejetora, pois se $y \in(R G) H, y=\sum_{h \in H} \alpha_{h} h$. Como cada $\alpha_{h} \in R G$, então $\alpha_{h}=\sum_{g \in G}\left(\alpha_{(g, h)}\right) g$. Tomando $\alpha=\sum_{g \in G, h \in H} \alpha_{(g, h)}(g, h) \in R(G \times H)$, obtemos $\varphi(\alpha)=y$. 


\subsection{Códigos}

Seja $A$ um conjunto finito, ao qual chamaremos alfabeto.

Definição 1.44. Um código de comprimento $n$ é um subconjunto próprio de $A^{n}$. Os elementos de um código serão chamados as palavras do código.

Definição 1.45. Sendo $|A|=s$, e tomando um código em $A^{n}$ com $M$ palavras, a taxa de informação de um código é dada por $R=\frac{\log _{s} M}{n}$.

Com o objetivo de medir a distância entre dois elementos do código, definimos:

Definição 1.46. Dado $x=\left(x_{1}, \ldots, x_{n}\right)$ e $y=\left(y_{1}, \ldots, y_{n}\right)$ dois elementos de $A^{n}, a$ distância de Hamming $d(x, y)$ entre $x$ e y é o número de posições nas quais $x$ e $y$ diferem, isto é,

$$
d(x, y)=\left|\left\{i ; x_{i} \neq y_{i}, 1 \leq i \leq n\right\}\right| .
$$

Note que a distância de Hamming é de fato uma distância, isto é, satisfaz os três axiomas de distância.

A distância mínima de um código $\mathcal{C} \subset A^{n}$ é o inteiro

$$
d(\mathcal{C})=\min \left\{d(x, y) ; x, y \in A^{n}, x \neq y\right\}
$$

Dado um alfabeto $A$, com $|A|=q, q$ potência de um número primo, o número máximo de palavras de um código de comprimento $n$ e distância mínima $d$ é denotado por $A_{q}(n, d)$. Um código sobre $A^{n}$ de distância mínima $d$ contendo $A_{q}(n, d)$ palavras é chamado um código ótimo. É objeto de estudo na Teoria de Códigos limitantes superiores e inferiores para $A_{q}(n, d)$. Alguns desses limitantes são:

Teorema 1.47. ([15], Teorema 4.5.6) Limitante de Singleton: $A_{q}(n, d)=q^{n-d+1}$.

Teorema 1.48. ([15], Teorema 4.5.4) Limitante de Gilbert-Varshamov: $A_{q}(n, d)=\frac{q^{n}}{\sum_{l=0}^{d-1}\left(\begin{array}{l}n \\ k\end{array}\right)(q-1)^{k}}$.

A classe de códigos que será alvo de nosso estudo é a seguinte: 
Definição 1.49. Dado $R$ um anel finito, um subconjunto próprio $C$ de $R^{n}$ é chamado código linear de comprimento $n$ sobre $R$ se $C$ é um $R$-submódulo de $R^{n}$.

Definição 1.50. Dado $\alpha=\left(\alpha_{1}, \ldots, \alpha_{n}\right) \in R^{n}$, o peso de $\alpha$ é dado por

$$
w(\alpha)=\left|\left\{i ; \alpha_{i} \neq 0,1 \leq i \leq n\right\}\right|
$$

O peso mínimo de um código $C$ é o inteiro

$$
w(C)=\min \{w(\alpha) ; \alpha \in C \backslash\{0\}\}
$$

Observe que o peso mínimo em um código linear é equivalente à distância mínima.

Definição 1.51. Um código linear não nulo $\mathcal{C}$ de $R^{n}$ é dito irredutível se não existe código não nulo $\mathcal{C}_{0}$ de $R^{n}$ contido propriamente em $\mathcal{C}$.

A classe de códigos lineares com a qual mais trabalharemos será:

Definição 1.52. Um código de peso constante é um código tal que todos os seus elementos não nulos têm mesmo peso.

Outro tipo de código linear é o seguinte:

Definição 1.53. Um código linear $C$ é dito código cíclico se, para toda palavra $\alpha=\left(\alpha_{1}, \ldots, \alpha_{n}\right) \in C$, a palavra $\left(\alpha_{n}, \alpha_{1}, \ldots, \alpha_{n-1}\right)$ está em $C$.

Quando as palavras do código são vistas como polinômios, temos que um código $C$ é cíclico se é um ideal de

$$
R_{n}=\frac{\mathcal{F}_{q}[x]}{\left\langle x^{n}-1\right\rangle} .
$$

Usando essa linguagem polinomial, temos o seguinte resultado.

Teorema 1.54. ([15], Teorema 7.4.1) Seja C um ideal em $R_{n}$.

1. Existe um único polinômio mônico $g(x)$ de grau mínimo em $C$. Este polinômio gera $C$, isto é, $C=\langle g(x)\rangle$, e é chamado polinômio gerador de $C$.

2. O polinômio gerador divide $x^{n}-1$ e, reciprocamente, se $p(x) \in R_{n}$ divide $x^{n}-1$, então $p(x)$ gera um código cíclico. 
3. Se $\operatorname{gr}(g(x))=r$, então $C$ tem dimensão $n-r$.

Como o polinômio gerador $g(x)$ divide $x^{n}-1$, então existe um polinômio $h(x)$ tal que

$$
x^{n}-1=g(x) h(x) .
$$

Tal polinômio $h(x)$ é chamado polinômio de checagem de $C$.

Definição 1.55. Um polinômio $e(x) \in R_{n}$ é dito idempotente $e m R_{n}$ se $e^{2}(x)=e(x)$.

Teorema 1.56. ([15], Teorema 7.4.9) Seja $C$ um código cíclico em $R_{n}$ com polinômio gerador $g(x)$ e polinômio de checagem $h(x)$, com $m d c(\operatorname{char} R, n)=1$. Então $g(x) e$ $h(x)$ são relativamente primos e, portanto, existem polinômios a $(x)$ e b $(x)$ para os quais

$$
a(x) g(x)+b(x) h(x)=1 .
$$

$O$ polinômio $e(x)=a(x) g(x) \bmod \left(x^{n}-1\right)$ tem por propriedades:

1. e $(x)$ é o único polinômio unidade em $C$, isto é, $p(x) e(x)=p(x)$, para todo $p(x) \in C$.

2. e $(x)$ é o único polinômio em $C$ que é idempotente e gera $C$.

Dado $R G$ anel de grupo, com $G$ um grupo cíclico de $n$ elementos gerado por $g$, um código $C$ de $R^{n}$ é cíclico se, e somente se, $\varphi(C)$ é um ideal de $R G$, com

$$
\begin{gathered}
\varphi: R^{n} \rightarrow R G \\
\alpha=\left(a_{0}, \ldots, a_{n-1}\right) \mapsto a_{0}+a_{1} g+\ldots+a_{n-1} g^{n-1}
\end{gathered}
$$

um isomorfismo de $R$-módulos. De fato, supondo $C$ código de $R^{n}$, usando o fato de $\operatorname{ser} \varphi$ isomorfismo de $R$-módulos, basta provar que, dado $\beta=\left(a_{0}, \ldots, a_{n-1}\right) \in C$, $g \varphi(\beta)$ está em $\varphi(C)$. Agora $g \varphi(\beta)=a_{n-1}+a_{0} g+\ldots+a_{n-2} g^{n-1}$. Como $C$ é cíclico, $\left(a_{n-1}, a_{0}, \ldots, a_{n-2}\right) \in C$, donde $g \varphi(\beta)=\varphi\left(a_{n-1}, a_{0}, \ldots, a_{n-2}\right) \in \varphi(C)$ e, portanto, $\varphi(C)$ é ideal de $R G$.

Reciprocamente, suponha $\varphi(C)$ ideal de $R G$. Dado então $\beta=\left(a_{0}, \ldots, a_{n-1}\right) \in C$, $g \varphi(\beta) \in \varphi(C)$, isto é, $a_{n-1}+a_{0} g+\ldots+a_{n-2} g^{n-1}=\varphi\left(a_{n-1}, a_{0}, \ldots, a_{n-2}\right) \in \varphi(C)$, donde $\left(a_{n-1}, a_{0}, \ldots, a_{n-2}\right) \in C$ e $C$ é cíclico. 
Como podemos ver um código cíclico como um ideal de $R_{n}$, podemos associar um ideal de $R G, G$ grupo cíclico com $n$ elementos gerado por $g$, com um código cíclico de $R_{n}$, associando $g$ a $x$. Com isso, podemos associar o polinômio gerador $g(x)$, bem como o idempotente $e(x)$, a um gerador de um ideal de $R G$, bem como a um idempotente $e$ de $R G$.

Um último resultado que gostaríamos de mencionar será muito importante no capítulo seguinte e por isso apresentaremos sua demonstração. É um resultado bastante conhecido da teoria de códigos e diz o seguinte:

Teorema 1.57. Seja $G=\left\{g_{1}, \cdots, g_{n}\right\}$ grupo abeliano de $n$ elementos, $\mathcal{F}_{q}$ corpo de $q$ elementos e $\mathcal{C}$ código não nulo de $\mathcal{F}_{q} G$ de dimensão $k$. Então a soma dos pesos dos elementos de $\mathcal{C}$ é

$$
\sum_{\alpha \in \mathcal{C}} w(\alpha)=n(q-1) q^{k-1}
$$

Prova: Seja $\alpha=\sum_{i=1}^{n} a_{i}(\alpha) g_{i}$ um elemento não nulo de $\mathcal{C}$. Então existe algum $j$, $1 \leq j \leq n$, tal que $a_{j}(\alpha)=m \neq 0$. Neste caso, tomando $g_{j}^{-1} \alpha \in \mathcal{C}$, neste elemento $a_{j}(\alpha)$ será o coeficiente de 1 . Também, para todo $j_{0}, 1 \leq j_{0} \leq n$, tomando o elemento $g_{j_{0}} g_{j}^{-1} \alpha \in \mathcal{C}$, nele $a_{j}(\alpha)$ será o coeficiente de $g_{j_{0}}$. Assim, dado um $j_{0}$, existe $\beta \in \mathcal{C}$ tal que $a_{j_{o}}(\beta) \neq 0$. Mais ainda, se $m \in \mathcal{F}_{q}, a_{j_{0}}\left(m\left(a_{j_{0}}(\alpha)\right)^{-1} \alpha\right)=m$, isto é, para todo $m \in \mathcal{F}_{q}, m \neq 0$, existe $\beta \in \mathcal{C}$ tal que $a_{j_{0}}(\beta)=m$. Com isso, fixado $\alpha \in \mathcal{C}$ tal que $a_{j_{0}}(\alpha)=m$, existe uma bijeção

$$
\begin{aligned}
\left\{\beta \in \mathcal{C} \mid a_{j_{0}}(\beta)=m \in \mathcal{F}_{q}^{*}\right\} & \longleftrightarrow\left\{\beta \in \mathcal{C} \mid a_{j_{0}}(\beta)=0\right\} \\
\beta & \mapsto \beta-\alpha .
\end{aligned}
$$

$\mathrm{E}$ assim $\left|\left\{\beta \in \mathcal{C} \mid a_{j_{0}}(\beta)=m \in \mathcal{F}_{q}^{*}\right\}\right|=\left|\left\{\beta \in \mathcal{C} \mid a_{j_{0}}(\beta)=0\right\}\right|$. Observe ainda que os conjuntos $\left\{\beta \in \mathcal{C} / a_{j_{0}}(\beta)=m \in \mathcal{F}_{q}\right\}$ são disjuntos para valores distintos de $m$. Portanto, $\mathcal{C}=\dot{\bigcup}_{m \in \mathcal{F}_{q}}\left\{\beta \mid a_{j}(\beta)=m\right\}$, conjuntos estes todos de mesma cardinalidade. E uma vez que $|\mathcal{C}|=q^{k}$, obtemos $\left|\left\{\beta \in \mathcal{C} \mid a_{j_{0}}(\beta)=m \in \mathcal{F}_{q}^{*}\right\}\right|=\frac{q^{k}}{q}=q^{k-1}$.

Observado isso, vejamos qual é a soma dos pesos dos elementos de $\mathcal{C}$. Dado $\alpha \in \mathcal{C}$, 
$w(\alpha)=\left|\left\{j \mid a_{j}(\alpha) \neq 0\right\}\right|$, então

$$
\begin{aligned}
\sum_{\alpha \in \mathcal{C}} w(\alpha) & =\sum_{\alpha \in \mathcal{C}}\left|\left\{j \mid a_{j}(\alpha) \neq 0\right\}\right| \\
& =\sum_{m \in \mathcal{F}_{q}^{*}} \sum_{\alpha \in \mathcal{C}}\left|\left\{j \mid a_{j}(\alpha)=m\right\}\right| \\
& =\sum_{1 \leq j \leq n} \sum_{m \in \mathcal{F}_{q}^{*}}\left|\left\{\alpha \in \mathcal{C} \mid a_{j}(\alpha)=m\right\}\right| \\
& =n(q-1) q^{k-1} .
\end{aligned}
$$

Para finalizar o capítulo, daremos duas definições que serão usadas ao longo do texto:

Definição 1.58. A função $\varphi$ de Euler, definida no conjunto dos números naturais, para cada $x \in \mathbb{N}$ é dada por:

$$
\begin{gathered}
\varphi(x)=|\{n \in \mathbb{N}|n<x| \operatorname{mdc}(n, x)=1\}|, x>1 \\
e \varphi(1)=1
\end{gathered}
$$

Definição 1.59. O delta de Kronecker é a notação $\delta_{i j}$ definida por:

$$
\delta_{i j}= \begin{cases}1, & \text { se } i=j \\ 0, & \text { se } i \neq j\end{cases}
$$




\section{Capítulo 2}

\section{Códigos Cíclicos de Peso Constante}

Neste capítulo apresentaremos condições que garantam que certos códigos cíclicos tenham peso constante baseados nos resultados de [19], mas fazendo uso aqui da Teoria de Anéis de Grupos, ao contrário do que é feito no artigo de Vega [19], no qual é utilizada a linguagem polinomial, sobretudo técnicas de sequências de recorrência linear. A vantagem é que, além da demonstração ter se tornado mais simples, o resultado se tornou mais geral. Os resultados serão obtidos em anéis de grupo da forma $\mathcal{F}_{q} G$, com $\mathcal{F}_{q}$ um corpo finito de $q$ elementos, e $C_{n}$ um grupo cíclico com $n$ elementos tal que $\operatorname{mdc}(q, n)=1$.

\subsection{Quando o código tem peso constante}

Nesta seção iremos generalizar um dos resultados de [19], em que o autor usou como ferramentas propriedades polinomiais. Trabalharemos aqui com códigos cíclicos vistos como ideais de anéis de grupos.

Sobre as hipóteses estabelecidas na introdução do capítulo o anel de grupo $\mathcal{F}_{q} G$ é semissimples. Dado $H$ um subgrupo de $G$, denotaremos por $\hat{H}$ o elemento $\frac{1}{|H|} \sum_{h \in H} h \in$ $G$. Observe que tal elemento é um idempotente.

De acordo com [2], temos a seguinte definição:

Definição 2.1. ([2],Definição 2.2) Um idempotente primitivo e em $\mathcal{F}_{q} G$ é dito essencial se $e \hat{H}=0$, para todo $H \neq 1, H$ subgrupo de $G$. 
Seja $e$ um idempotente primitivo em $\mathcal{F}_{q} G$ não essencial. Considere $\mathcal{H}_{e}=\{H<G ; \hat{H} e=e\}$. Note que $\widehat{H_{1} H_{2}}=\hat{H}_{1} \hat{H}_{2}$, donde se $H_{1}, H_{2} \in \mathcal{H}_{e}, \widehat{H_{1} H_{2}} e=e$.

Seja $H_{e}:=\prod_{H \in \mathcal{H}} H$. Então $H_{e} \in \mathcal{H}_{e}$, e é o maior subgrupo $H$ de $G$ tal que $e \hat{H}=e$.

Dado um idempotente $k$, ou $e k=0$ ou $e k=e$. De fato, se $e k=j \neq 0$, então $j$ seria idempotente, e $e j=e e k=j$. Daí, $e=e-j+j$, $\operatorname{com}(e-j)(e-j)=e-2 j+j=e-j$, e $(e-j) j=j-j=0$, contradizendo o fato de ser $e$ primitivo. Assim, se $K \not H_{e}$, $e \hat{K}=0$, pois senão, como $\widehat{K H_{e}}$ é idempotente, $e=e \hat{K}=e \widehat{K H_{e}}$, o que seria uma contradição com a maximalidade de $H_{e}$. Disto $e \hat{K}=e$ se, e somente se, $K \subseteq H_{e}$.

Como visto em [2], Observação 2.1 e Proposição 3.3, temos:

Lema 2.2. Seja e um idempotente primitivo de $\mathcal{F}_{q} G$ e $H_{e}$ como definido acima. A função:

$$
\begin{aligned}
\Psi: \mathcal{F}_{q} G \hat{H}_{e} & \rightarrow \mathcal{F}_{q}\left(\frac{G}{H_{e}}\right) \\
g \hat{H}_{e} & \mapsto \bar{g} \\
\sum_{g \in G} \alpha_{g} g \hat{H}_{e} & \mapsto \sum_{g \in G} \alpha_{g} \bar{g}
\end{aligned}
$$

é um isomorfismo de anéis, com $\Psi(e)$ idempotente essencial.

Prova: De fato, temos primeiramente $\frac{G}{H_{e}} \simeq G e$, pois a aplicação $\phi: G \rightarrow G e$, dada por $g \mapsto g e$ é homomorfismo sobrejetor de grupos com $\operatorname{Ker}(\phi)=H_{e}$, pois se $h \in H_{e}$, $h e=h \hat{H}_{e} e=\hat{H}_{e} e=e$, e se $a e=e,\langle a\rangle \subset H_{e}$. Como Ge gera $R G e$ sobre $R$ segue que $\Psi$ é isomorfismo de anéis. Como $e \hat{H}_{e}=e, e \in \mathcal{F}_{q} G \hat{H}_{e}$.

Seja $L<\frac{G}{H_{e}}$. Então $L=\frac{L^{\prime}}{H_{e}}$, para algum $L^{\prime}$ subgrupo de $G$ tal que $H_{e} \subseteq L^{\prime} \subseteq G$. Suponha $L \neq 1$, o que ocorre se, e somente se $L^{\prime} \neq H_{e}$. Analisemos $\Psi(e) \hat{L}=\Psi\left(e \hat{L}^{\prime}\right)$. Se $L^{\prime} \supset H_{e}, \hat{L}^{\prime} \hat{H}_{e}=\hat{L}^{\prime} \mapsto \frac{1}{\left|L^{\prime}\right|} \sum_{h \in L / H_{e}} \bar{h}$. Como temos $\left|H_{e}\right|$ cópias de $\bar{h}$ em $\frac{G}{H_{e}}$, então

$$
\Psi\left(\hat{L}^{\prime}\right)=\frac{\left|H_{e}\right|}{\left|L^{\prime}\right|} \sum_{\bar{h} \in L^{\prime} / H_{e}} \bar{h}=\frac{1}{\left|\frac{L^{\prime}}{H_{e}}\right|} \sum_{\bar{h} \in \frac{L^{\prime}}{H_{e}}} \bar{h}
$$

donde

$$
\Psi(e) \hat{L}=\Psi(e)\left(\frac{\widehat{L}}{H_{e}}\right)=\Psi(e) \Psi\left(\hat{L}^{\prime}\right)=\Psi\left(e \hat{L}^{\prime}\right)=0
$$

pois $L^{\prime}$ não está contido em $H_{e}$ e portanto $\Psi(e)$ é um idempotente primitivo essencial de $\mathcal{F}_{q}\left(\frac{G}{H_{e}}\right)$. 
Lema 2.3. ([2], Proposição 2.3) Seja e um idempotente primitivo em $\mathcal{F}_{q} G$, para $G$ um grupo abeliano finito. Então e é essencial se, e somente se $G \simeq G e$ via o homomorfismo de grupos

$$
\begin{gathered}
\pi: G \rightarrow G e \\
g \mapsto g e .
\end{gathered}
$$

Prova: O homomorfismo de grupos $\pi$ é claramente sobrejetor. Suponha $e$ não essencial. Então existe $H \neq 1$ subgrupo de $G$ tal que $e \hat{H}=e$. Seja $h \in H, h \neq 1$. Então $h e=h \hat{H} e=\hat{H} e=e$ e, portanto, $\pi(h)=e$, donde $\pi$ não é injetora, não sendo assim isomorfismo. Por outro lado se $\pi$ não é isomorfismo, ou seja, se $\pi$ não é injetora, então existe $h \neq 1 \in G$ tal que $\pi(h)=e$, donde, para todo $i, h^{i} e=e$ e, portanto, para $H=\langle h\rangle, e \hat{H}=e$, e $e$ não é essencial.

No artigo de Vega [19], é muito usada a noção de ordem e quase ordem de um polinômio, definições essas que podem ser encontradas em [10]. Comecemos discutindo o equivalente da definição de ordem na linguagem de anéis de grupo.

Definição 2.4. Assuma $C_{n}=<g>$ o grupo cíclico com $n$ elementos, $\mathcal{F}_{q}$ corpo finito com $q$ elementos e e um idempotente primitivo em $\mathcal{F}_{q} C_{n}$. A ordem de ge, que denotaremos por o(ge), é o menor $m$ inteiro positivo tal que $(\mathrm{ge})^{m}=1$, isto é, é a ordem de ge em $C_{n}$ e. Observe que a ordem de ge é igual a ordem de $p(x)$, em que $p(x)$ é polinômio irredutivel de $\mathcal{F}_{q}[x]$ tal que $\mathcal{F}_{q} C_{n} e \simeq \frac{\mathcal{F}_{q}[x]}{\langle p(x)\rangle}, p(x)$ dividindo $x^{n}-1$.

Vejamos agora o que é a quase ordem na linguagem de anéis de grupos.

Sabemos que, tomando e idempotente primitivo em $\mathcal{F}_{q} C_{n}, \mathcal{F}_{q} C_{n}$ e é um corpo. Considere os conjuntos $\mathcal{F}_{q}^{*}$ e e $C_{n}$ e. Ambos são subgrupos de $\left(\mathcal{F}_{q} C_{n} e\right)^{*}=U\left(\mathcal{F}_{q} C_{n} e\right)$, donde $C_{n} e \cap \mathcal{F}_{q} e \subseteq U\left(\mathcal{F}_{q} C_{n} e\right)$. Pelo Teorema1.10, $\left|C_{n} e \cap \mathcal{F}_{q}^{*} e\right|=\operatorname{mdc}(o(g e), q-1)$. Mas o único subgrupo cíclico de $C_{n}$ e de ordem $m d c(o(g e), q-1)$ é $\left\langle(g e)^{o(g e) / m d c(o(g e), q-1)}\right\rangle$, donde $C_{n} e \cap \mathcal{F}_{q}^{*} e=\left\langle(g e)^{o(g e) / m d c(o(g e), q-1)}\right\rangle e$, portanto, $(g e)^{o(g e) / m d c(o(g e), q-1)} \in \mathcal{F}_{q} e$, $\operatorname{digamos}(g e)^{(o(g e) / m d c(o(g e), q-1)}=k e, k \in \mathcal{F}_{q}^{*} \cdot E o(g e) / m d c(o(g e), q-1)$ é o menor inteiro $\lambda$ tal que $(\mathrm{ge})^{\lambda} \in \mathcal{F}_{q}^{*}$ e. Com isso, temos:

Definição 2.5. Com a notação acima, a quase ordem de ge, que denotaremos por qord $(g e)$, é dada por qord $(g e)=\frac{o(g e)}{m d c(o(g e), q-1)}$. 
Antes do nosso principal resultado desta seção, façamos um último resultado sobre um código em $\mathcal{F}_{q} C_{n}$ :

Lema 2.6. Se $\mathcal{C}$ é um código de $\mathcal{F}_{q} C_{n}$ de peso constante, então $\mathcal{C}$ é irredutível.

Prova: Tome $\mathcal{C}$ um código de $\mathcal{F}_{q} C_{n}$ de peso constante e suponha que ele não seja irredutivel. Seja $\mathcal{C}_{0} \neq 0$ um código contido em $\mathcal{C}$ e diferente de $\mathcal{C}$. Suponha $\operatorname{dim}_{\mathcal{F}_{q}} \mathcal{C}=k$, $e \operatorname{dim} \mathcal{F}_{q} \mathcal{C}_{0}=k_{0}$. Então $k_{0}<k$. Se $\mathcal{C}$ tem peso constante, dado $0 \neq \alpha \in \mathcal{C}$, usando a fórmula de soma de pesos, e sabendo que existem $q^{k}-1$ elementos não nulos em $\mathcal{C}$, concluímos que $\omega(\alpha)=\frac{q^{k-1}(q-1) n}{q^{k}-1}$. Como $\mathcal{C}_{0}$ também terá peso constante, então, para $\alpha \in \mathcal{C}_{0}, \omega(\alpha)=\frac{q^{k_{0}-1}(q-1) n}{q^{k_{0}-1}}$. Assim, fixando $0 \neq \alpha \in \mathcal{C}_{0} \subset \mathcal{C}, \frac{q^{k-1}(q-1) n}{q^{k}-1}=\omega(\alpha)=$ $\frac{q^{k_{0}-1}(q-1) n}{q^{k_{0}}-1}$, o que é absurdo, uma vez que manipulando a igualdade acima concluiríamos que $q^{k}=q^{k_{0}}$, o que não ocorre uma vez que $k \neq k_{0}$, e, portanto, o código deve ser irredutivel.

Sendo assim, para determinar os códigos de peso constante em $\mathcal{F}_{q} C_{n}$, basta que analisemos seus códigos irredutíveis. Temos então:

Teorema 2.7. Sejam $\mathcal{F}_{q}$ corpo com $q$ elementos, $n$ um inteiro positivo com $\operatorname{mdc}(q, n)=1, C_{n}=<g>$ grupo cíclico com $n$ elementos e $\mathcal{F}_{q} C_{n}$ o anel de grupo de $C_{n}$ sobre $\mathcal{F}_{q}$. Considere e um idempotente primitivo de $\mathcal{F}_{q} C_{n}$ e $\mathcal{C}=\mathcal{F}_{q} C_{n}$ e o respectivo código irredutível. Seja $\operatorname{dim}_{\mathcal{F}_{q}} \mathcal{C}=k$. São equivalentes:

1. $\mathcal{C}$ tem peso constante;

2. Todo elemento não nulo de $\mathcal{C}$ tem peso $\frac{q^{k-1}(q-1) n}{q^{k}-1}$;

3. Existe um elemento de $\mathcal{C}$ cujo peso é $\frac{q^{k-1}(q-1) n}{q^{k}-1}$;

4. $\left(\mathcal{F}_{q} C_{n} e\right)^{*}=\mathcal{F}_{q}^{*} e C_{n} e$.

Prova: $(1 \Longrightarrow 2)$ : Segue do fato que para um código abeliano $\mathcal{C}$ nas condições do teorema vale, pelo Teorema 1.57, a seguinte fórmula de soma de pesos:

$$
\sum_{\alpha \in \mathcal{C}} \omega(\alpha)=q^{k-1}(q-1) n
$$

e do fato que existem $q^{k}-1$ elementos não nulos no código. 
$(2 \Longrightarrow 3)$ Imediata.

(3 $\Longrightarrow 4)$ Assuma inicialmente e essencial. Neste caso, $\left|C_{n} e\right|=n$, de acordo com o Lema 2.3. Pelo Lema 1.10, $\left|C_{n} e \cap \mathcal{F}_{q}^{*} e\right|=\operatorname{mdc}\left(\left|C_{n} e\right|,\left|\mathcal{F}_{q}^{*} e\right|\right)$, bem como $\left|C_{n} e \mathcal{F}_{q}^{*} e\right|=$ $\operatorname{mmc}\left(\left|C_{n} e\right|,\left|\mathcal{F}_{q}^{*} e\right|\right)$. Seja $\mu=\operatorname{mdc}\left(\left|C_{n} e\right|,\left|\mathcal{F}_{q}^{*} e\right|\right)$, e $t=n / \mu$. Só há um subgrupo de ordem $\mu$ em $\left(\mathcal{F}_{q} C_{n} e\right)^{*}$, que é $\left\langle g^{t} e\right\rangle$. Como $C_{n} e \cap \mathcal{F}_{q}^{*}$ e também tem tal ordem, então $C_{n} e \cap \mathcal{F}_{q}^{*} e=\left\langle g^{t} e\right\rangle$. Logo $g^{t} e=\lambda e$, para algum $\lambda \in \mathcal{F}_{q}^{*}$. Seja $\alpha$ o elemento de peso $\frac{q^{k-1}(q-1) n}{q^{k}-1}$. Como e é a unidade de $\mathcal{F}_{q} C_{n} e, g^{t} \alpha=g^{t} e \alpha=\lambda e \alpha=\lambda \alpha$. Como

$$
\alpha=\alpha_{0}+\alpha_{1} g+\ldots+\alpha_{n-1} g^{n-1}
$$

temos

$$
\alpha g^{t}=\alpha_{0} g^{t}+\alpha_{1} g^{t+1}+\ldots+\alpha_{n-t}+\alpha_{n-t+1} g+\ldots+\alpha_{n-1} g^{n+t-1}=\lambda \alpha .
$$

Disto $\alpha_{0}=\alpha_{t} \lambda$, bem como $\alpha_{t}=\alpha_{2 t} \lambda$, isto é, $\alpha_{2 t}=\alpha_{t} \lambda^{-1}=\alpha_{0} \lambda^{-2}$ e, de um modo geral, $\alpha_{i+k t}=\alpha_{i} \lambda^{-k}$, para todos $0 \leq i \leq t-1,0 \leq k \leq \mu-1$.

Considere $\beta=\alpha_{0}+\alpha_{1} g+\ldots+\alpha_{t-1} g^{t-1}$. Então

$$
\begin{aligned}
\alpha & =\beta+\lambda^{-1} \beta g^{t}+\lambda^{-2} \beta g^{2 t}+\ldots+\lambda^{-(\mu-1)} \beta g^{(\mu-1) t} \\
& =\sum_{i=1}^{\mu-1} \lambda^{-i} g^{i t} \beta .
\end{aligned}
$$

com $\operatorname{supp}\left(\lambda^{-i} g^{i t} \beta\right) \cap \operatorname{supp}\left(\lambda^{-j} g^{j t} \beta\right)=\emptyset$, se $i \neq j$. Disto

$$
\omega(\alpha)=\omega(\beta)+\ldots+\omega\left(\lambda^{\mu-1} \beta g^{(\mu-1) t}\right)=\mu \omega(\beta)
$$

e, assim, $\mu=\operatorname{mdc}\left(\left|C_{n} e\right|,\left|\mathcal{F}_{q}^{*} e\right|\right)$ divide $\frac{q^{k-1}(q-1) n}{q^{k}-1}$, isto é, $\operatorname{mdc}(n, q-1)$ divide $\frac{q^{k-1}(q-1) n}{q^{k}-1}$. Como $m d c\left(q^{k-1}, \operatorname{mdc}(n, q-1)\right)=1$, pois por hipótese $m d c(n, q)=1$, segue que $\operatorname{mdc}(n, q-1)$ divide $\frac{(q-1) n}{q^{k}-1}$ e, portanto, $q^{k}-1$ divide $\frac{(q-1) n}{m d c(n, q-1)}=\operatorname{mmc}(q-1, n)=$ $\left|\mathcal{F}_{q}^{*} e C_{n} e\right|$, isto é, $\left|\left(\mathcal{F}_{q} C_{n} e\right)^{*}\right|$ divide $\left|\mathcal{F}_{q}^{*} e C_{n} e\right|$. Como $\mathcal{F}_{q}^{*} e C_{n}$ e é subgrupo de $\left(\mathcal{F}_{q} C_{n} e\right)^{*}$, então $\left(\mathcal{F}_{q} C_{n} e\right)^{*}=\mathcal{F}_{q}^{*} e C_{n}$ e. Isto termina o caso em que e é essencial.

Suponha agora e não essencial. Tome então $H_{e}$ o maior subgrupo de $C_{n}$ tal que e $H_{e}=$ e. Como vimos anteriormente $\mathcal{F}_{q} C_{n} \hat{H}_{e} \simeq \mathcal{F}_{q} \frac{C_{n}}{H_{e}}$ via o isomorfismo $\psi$ tal que $\Psi\left(g \hat{H}_{e}\right)=\bar{g}$. Considere $\left|H_{e}\right|=d_{1},\left|\frac{C_{n}}{H_{e}}\right|=d_{2}$, com $H_{e}=\left\langle g^{d_{2}}\right\rangle$. Seja $\alpha \in \mathcal{F}_{q} C_{n} \hat{H}_{e} d e$ peso $\frac{q^{k-1}(q-1) n}{q^{k}-1}$. Então, como $\alpha \hat{H}_{e}=\alpha$, como antes, temos

$$
\begin{aligned}
\alpha & =\left(\alpha_{0}+\alpha_{1} g+\ldots+\alpha_{d_{2}-1} g^{d_{2}-1}\right)+\alpha_{0} g^{d_{2}}+\ldots \\
& =\left(\alpha_{0}+\alpha_{1} g+\ldots+\alpha_{d_{2}-1} g^{d_{2}-1}\right) \hat{H}_{e}
\end{aligned}
$$


e assim

$$
\omega(\alpha)=\omega\left(\alpha_{0}+\alpha_{1} g+\ldots+\alpha_{d_{2}-1} g^{d_{2}-1}\right)\left|H_{e}\right|
$$

Agora

$$
\Psi(\alpha)=\alpha_{0}+\alpha_{1} \bar{g}+\ldots+\alpha_{d_{2}-1} \bar{g}^{d_{2}-1},
$$

$\operatorname{logo} \omega(\Psi(\alpha))=\frac{\omega(\alpha)}{d_{1}}$, e daí $\omega(\Psi(\alpha))=\left(\frac{q^{k-1}(q-1) n}{q^{k}-1}\right) / d_{1}=\frac{q^{k-1}(q-1) d_{2}}{q^{k}-1}$, com $d_{2}=\left|\frac{C_{n}}{H_{e}}\right|$. $\operatorname{Em} \mathcal{F}_{q} \frac{C_{n}}{H_{e}} \Psi(e), \Psi(e)$ é essencial e, neste caso, $\mathcal{F}_{q}^{*} \Psi(e) \frac{C_{n}}{H_{e}} \Psi(e)=\left(\mathcal{F}_{q} \frac{C_{n}}{H_{e}} \Psi(e)\right)^{*}$. Deste fato, um elemento qualquer é da forma $\lambda \bar{g}^{i} \Psi(e)$, logo, voltando pela $\Psi^{-1}$ (pois $\Psi$ é isomorfismo), segue que os elementos de $\left(\mathcal{F}_{q} C_{n}\right.$ e) ${ }^{*}$ são da forma $\lambda g^{i}$ e e temos o resultado também neste caso.

$(4 \Longrightarrow 1):$ Como $\left(\mathcal{F}_{q} C_{n} e\right)^{*}=\mathcal{F}_{q}^{*} e C_{n}$ e, os elementos não nulos de $\mathcal{F}_{q} C_{n}$ e são da forma $\lambda g^{i}$ e, com $\lambda \in \mathcal{F}_{q}^{*}$, donde todos têm o mesmo peso.

Uma consequência imediata deste resultado é o seguinte:

Corolário 2.8. Se $\mathcal{C}$ é um código de $\mathcal{F}_{q} C_{n}(\operatorname{com} \operatorname{mdc}(n, q)=1)$, então $\mathcal{C}$ tem peso constante se, e somente se, $\mathcal{C}=\{0\} \cup\left\{k g^{i} e \mid k \in \mathcal{F}_{q}^{*}, g^{i} \in C_{n}\right\}$.

Prova: Do teorema anterior, um código de $\mathcal{F}_{q} C_{n}$ tem peso constante se, e somente se, $\left(\mathcal{F}_{q} C_{n} e\right)^{*}=\mathcal{F}_{q}^{*} e C_{n} e$, isto é, se, e somente se, $\mathcal{C}=\{0\} \cup\left\{k g^{i} e \mid k \in \mathcal{F}_{q}^{*}, g^{i} \in C_{n}\right\}$.

A partir do teorema obtemos o seguinte resultado encontrado no artigo de Vega em [19].

Corolário 2.9 ([19], Teorema 9). Seja $\mathcal{F}_{q}$ um corpo com $q$ elementos, $n=\lambda \frac{q^{k}-1}{q-1}$, com $\lambda$ dividindo $(q-1)$, e $\mathcal{C}$ um código cíclico sobre $\mathcal{F}_{q}$ de comprimento $n$ e dimensão $k$, gerado por $g(x)$ e com polinômio de checagem $h(x)$. Então qord $(g e)=\frac{q^{k}-1}{q-1}$ se, $e$ somente se, $w(g e)=\lambda\left(q^{k-1}\right)$.

Prova: De fato, qord $(h(x))=\frac{o(g e)}{m d c(o(g e), q-1)}$ é igual a $\frac{q^{k}-1}{q-1}$ se, e somente se $\frac{o(g e)(q-1)}{m d c(o(g e), q-1)}=q^{k}-1$, isto é, se, e somente se, $\left(\mathcal{F}_{q} C_{n} e\right)=\mathcal{F}_{q}^{*} e C_{n} e$, o que ocorre de acordo com o Teorema 2.7 se, e somente se, $\mathcal{C}$ tem peso constante. Assim, isto ocorre se, e somente se, $\omega(g e)=\frac{q^{k-1}(q-1) n}{q^{k}-1}$. Como $\lambda=\frac{n(q-1)}{q^{k}-1}$, isso ocorre se, e somente se, $\omega(g e)=\lambda\left(q^{k-1}\right)$. 


\subsection{Quantidade de códigos de peso constante}

Agora que já sabemos quais são os códigos de peso constante, nosso objetivo aqui é determinar a quantidade de códigos de peso constante de dimensão $k$ e comprimento $n$ em $\mathcal{F}_{q} C_{n}, C_{n}=\langle g\rangle$ grupo cíclico de $n$ elementos com $\operatorname{mdc}(n, q)=1$, usando para isso o Teorema 2.7, bem como propriedades de idempotentes essenciais encontradas em [2], generalizando assim o Teorema 12 de [19]. Para isso, começaremos com uma análise dos idempotentes primitivos de $\mathcal{F}_{q} C_{n}$.

Seja $n=p_{1}^{r_{1}} p_{2}^{r_{2}} \cdots p_{t}^{r_{t}}$. Então $C_{n}=P_{1} \times \cdots P_{t}$, em que $P_{i}$ é o $p_{i}$-Sylow de $C_{n}$. Como $C_{n}$ é cíclico, seus subgrupos são cíclicos e, em particular, cada $P_{i}$ é cíclico, donde existe $K_{i}$ subgrupo minimal de $P_{i}$, para cada $i, 1 \leq i \leq t$. Então

$$
e_{0}=\left(1-\hat{K}_{1}\right)\left(1-\hat{K}_{2}\right) \cdots\left(1-\hat{K}_{t}\right)
$$

é um idempotente e um idempotente primitivo e de $\mathcal{F}_{q} C_{n}$ é essencial se, e somente se, $e e_{0}=e$. De fato, se e é essencial, $e \hat{H}=0$, para todo $H$ subgrupo próprio de $C_{n}$, donde $e e_{0}=e\left(1-\hat{K}_{1}\right)\left(1-\hat{K}_{2}\right) \cdots\left(1-\hat{K}_{t}\right)=\left(e-e \hat{K}_{1}\right)\left(1-\hat{K}_{2}\right) \cdots\left(1-\hat{K}_{t}\right)=$ $(e-0)\left(1-\hat{K}_{2}\right) \cdots\left(1-\hat{K}_{t}\right)=\cdots=e$. E se e não for essencial, existe $H \neq 1$ subgrupo próprio de $C_{n}$ com $e \hat{H}=e$. Como $H$ é um subgrupo não trivial de $C_{n}$, existe $i, 1 \leq i \leq t$, tal que $K_{i} \subset H$. Assim ee $e_{0}=e \hat{H} e_{0}=0$, pois $\hat{H}\left(1-\hat{K}_{i}\right)=0$. Disto, ao decompor e e como soma de idempotentes primitivos dois a dois ortogonais, digamos $e_{0}=e_{1}+e_{2}+\cdots+e_{m}$, uma vez que teremos $e_{0} e_{i}=e_{i}$, para todos $1 \leq i \leq m$, o que implicará que cada $e_{i}$ é essencial, bem como todo essencial é um dos $e_{i}$ pois $e_{0}=e_{i}+\left(e_{0}-e_{i}\right)$, obtemos $e_{0}=\sum_{\text {e essencial }} e$, isto é, $e_{0}$ é soma de todos os idempotentes essenciais em $\mathcal{F}_{q} C_{n}$.

Em [2], encontramos o seguinte resultado:

Teorema 2.10. ([2], Teorema 4.2) Seja $C_{n}$ um grupo cíclico com $n$ elementos e com gerador $g$ e seja $m$ o menor inteiro positivo tal que $n \mid\left(q^{m}-1\right)$. Então:

1. $\operatorname{dim}\left(\mathcal{F}_{q} C_{n}\right) e_{0}=\varphi(n)$, em que $\varphi$ denota a função de Euler.

2. Existem precisamente $\frac{\varphi(n)}{m}$ idempotentes essenciais em $\mathcal{F}_{q} C_{n}$.

Com esse resultado e o Teorema 2.7, temos o seguinte: 
Teorema 2.11. Sejam $\mathcal{F}_{q}$ um corpo com $q$ elementos, $n$ um inteiro positivo com $\operatorname{mdc}(q, n)=1, C_{n}=<g>$ grupo cíclico com $n$ elementos e $\mathcal{F}_{q} C_{n}$ o anel de grupo de $C_{n}$ sobre $\mathcal{F}_{q}$. O número de códigos de peso constante de comprimento $n$ e dimensão $k e ́$

$$
\sum_{d \mid n} \delta_{\left(q^{k}-1, m m c(d, q-1)\right)} \frac{\varphi(d)}{k},
$$

em que $\varphi$ é a função de Euler e $\delta$ é a função delta de Kronecker.

Prova: Pelo Teorema 2.7, dado e idempotente primitivo de $\mathcal{F}_{q} C_{n}, \mathcal{F}_{q} C_{n}$ e de dimensão $k$ tem peso constante se, e somente se, $\left(\mathcal{F}_{q} C_{n} e\right)^{*}=\mathcal{F}_{q}^{*} e C_{n} e$, isto é, se e somente se, sendo $d=o(g e)$ e $q^{k}-1=\operatorname{mmc}(q-1, d)$.

Seja $d \mid n$ com $q^{k}-1=m m c(q-1, d)$. Considere $C_{d}$ grupo cíclico com d elementos, e tome os idempotentes primitivos essenciais em $\mathcal{F}_{q} C_{d}$, que são, de acordo com o Lema 2.2 os idempotentes primitivos de $\mathcal{F}_{q} C_{n}$ com o $($ ge $)=d$, temos, como $e_{0}=\sum_{\text {e essencial }} e, e$ usando o Teorema 2.10, sendo $l$ o número de essenciais de $\mathcal{F}_{q} C_{d}$ :

$$
\begin{aligned}
\mathcal{F}_{q} C_{d} e_{0} & =\bigoplus_{\text {e essencial }} \mathcal{F}_{q} C_{d} e \\
\Rightarrow \operatorname{dim}_{\mathcal{F}_{q}} \mathcal{F}_{q} C_{d} e_{0} & =\sum_{\text {e essencial }} \operatorname{dim}_{\mathcal{F}_{q}} \mathcal{F}_{q} C_{d} e \\
\Rightarrow \varphi(d) & =l \cdot k \\
\Rightarrow l & =\frac{\varphi(d)}{k} .
\end{aligned}
$$

Como o número de idempotentes essenciais determina a quantidade de códigos distintos de peso constante, temos para um tal d que a quantidade de códigos de peso constante é $\frac{\varphi(d)}{k}$. Somando as quantidades para cada $d \mid n$ que satisfaz $q^{k}-1=m m c(q-1, d)$, obtemos o resultado.

\subsection{Códigos de dois pesos}

Nesta seção construiremos códigos de dois pesos, usando para isso o que sabemos sobre códigos de peso constante pela seção anterior.

Considere o anel de grupo $\mathcal{F}_{q}\left(C_{q^{m}-1} \times C_{q^{m}-1}\right)=\left(\mathcal{F}_{q}\langle g\rangle\right)\langle h\rangle$, em que g (bem como h) gera $C_{q^{m}-1}$. Seja e um idempotente essencial de $\mathcal{F}_{q} C_{q^{m}-1}$, o qual representaremos 
por e $(g)$ em $\mathcal{F}_{q}\langle g\rangle$ e por e $(h)$ em $\mathcal{F}_{q}\langle h\rangle$, e suponha que $\mathcal{F}_{q} C_{q^{m}-1}$ e tenha peso constante. Seja

$$
\hat{g}=\frac{1}{q^{m}-1} \sum_{0 \leq i \leq q^{m}-2} g^{i}
$$

bem como

$$
\hat{h}=\frac{1}{q^{m}-1} \sum_{0 \leq i \leq q^{m}-2} h^{i} .
$$

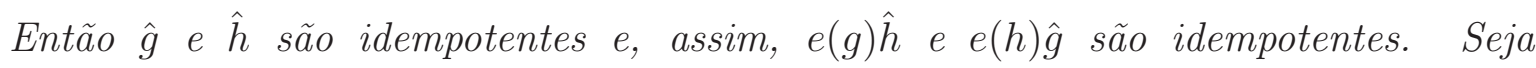
$e_{0}=e(g) \hat{h}+e(h) \hat{g}$. Analisemos então o código

$$
\mathcal{F}_{q}\left(C_{q^{m}-1} \times C_{q^{m}-1}\right) e_{0}
$$

Queremos saber quais os pesos dos elementos não nulos deste código. Para isso, verifiquemos antes quais são os elementos de tal código.

Teorema 2.12. Com a notação introduzida acima, os elementos não nulos de $\mathcal{F}_{q}\left(C_{q^{m}-1} \times C_{q^{m}-1}\right) e_{0}$ são da forma $g^{i} e(g) \hat{h}, 1 \leq i \leq q^{m}-1, h^{i} e(h) \hat{g}, 1 \leq i \leq q^{m}-1$, e $g^{t} h^{s} e_{0}, 1 \leq t, s \leq q^{m}-1$.

Prova: Considere $L=\{0\} \cup\left\{g^{i} e(g) \hat{h}\right\} \cup\left\{h^{j} e(h) \hat{g}\right\} \cup\left\{g^{t} h^{s} e_{0}\right\}$. Vejamos que $L=\left\langle e_{0}\right\rangle$.

$A$ inclusão $L \subseteq\left\langle e_{0}\right\rangle$ é verdadeira, pois uma vez que e $(h)$ e e $(g)$ são essenciais em $\langle h\rangle$ e $\langle g\rangle$, respectivamente, temos $e(h) \cdot \hat{h}=0=e(g) \cdot \hat{g}$, e devido a isso é imediato que os elementos da forma $g^{i} e(g) \hat{h}, 1 \leq i \leq q^{m}-1$, bem como os elementos da forma $h^{i} e(h) \hat{g}, 1 \leq i \leq q^{m}-1$, além do 0 , estão em $\mathcal{F}_{q}\left(C_{q^{m}-1} \times C_{q^{m}-1}\right) e_{0}$. Já para elementos da forma $g^{t} h^{s} e_{0}$, com $1 \leq t, s \leq q^{m}-1$, a inclusão é imediata.

Para verificar a inclusão $\left\langle e_{0}\right\rangle \subseteq L$, uma vez que $e_{0} \in L$, basta provar que $L$ é ideal. Vejamos inicialmente que $L$ é fechado para a soma.

Comecemos analisando a soma $g^{t} h^{s} e_{0}+g^{t_{1}} h^{s_{1}} e_{0}$, quando esta é não nula. Temos

$$
\begin{aligned}
g^{t} h^{s} e_{0}+g^{t_{1}} h^{s_{1}} e_{0} & =g^{t} h^{s}(e(g) \hat{h}+e(h) \hat{g})+g^{t_{1}} h^{s_{1}}(e(g) \hat{h}+e(h) \hat{g}) \\
& =g^{t} e(g) \hat{h}+g^{t_{1}} e(g) \hat{h}+h^{s} e(h) \hat{g}+h^{s_{1}} e(h) \hat{g} \\
& =\left(\left(g^{t}+g^{t_{1}}\right) e(g)\right) \hat{h}+\left(\left(h^{s}+h^{s_{1}}\right) e(h)\right) \hat{g} .
\end{aligned}
$$

Note que o elemento $\left(g^{t}+g^{t_{1}}\right) e(g)$ é um elemento do código $\mathcal{F}_{q} C_{q^{m}-1} e(g)$. Suponha inicialmente que $g^{t}+g^{t_{1}}$ é diferente de zero. Como $\mathcal{F}_{q} C_{q^{m}-1} e(g)$ tem peso constante, temos, pelo Teorema 2.7 (que pode ser aplicado aqui pois $\operatorname{mdc}\left(q, q^{m}-1\right)=1$ ), 
$\left(\mathcal{F}_{q} C_{q^{m}-1} e(g)\right)^{*}=\mathcal{F}_{q}^{*} e(g) C_{q^{m}-1} e(g) . \quad$ Como e $(g)$ é essencial, pelo Lema 2.3, $C_{q^{m}-1} e(g) \simeq C_{q^{m}-1}$, donde $\left|C_{q^{m}-1} e(g)\right|=q^{m}-1$ e, assim, $\left|\mathcal{F}_{q} C_{q^{m}-1} e(g)\right|=q^{m}-1$, donde $\left|\left(\mathcal{F}_{q} C_{q^{m}-1} e(g)\right)^{*}\right|=q^{m}-1$. Como e $(g)$ é essencial, $g^{i} e(g) \neq g^{j} e(g)$, se $i \neq j$, donde $\left|\left\{g^{i} e(g)\right\}\right|=q^{m}-1 e$, daí, $\left(g^{t}+g^{t_{1}}\right) e(g)=g^{i} e(g)$, para algum $i, 1 \leq i \leq q^{m}-1$.

O mesmo vale para $\left(h^{s}+h^{s_{1}}\right) e(h)$, caso a soma $h^{s}+h^{s_{1}}$ seja diferente de zero.Portanto

$$
\begin{aligned}
g^{t} h^{s} e_{0}+g^{t_{1}} h^{s_{1}} e_{0} & =\left(\left(g^{t}+g^{t_{1}}\right) e(g)\right) \hat{h}+\left(\left(h^{s}+h^{s_{1}}\right) e(h)\right) \hat{g} \\
& =g^{i} e(g) \hat{h}+h^{j} e(h) \hat{g}=g^{i} h^{j} e_{0},
\end{aligned}
$$

pois $\hat{g}=g^{i} \hat{g}$, e $\hat{h}=h^{i} \hat{h}$.

Caso alguma dessas somas dê igual a 0 , recaímos numa soma em $\langle e(g)\rangle($ ou $\langle e(h)\rangle)$, que é ideal, sendo portanto fechado para a soma.

Por fim, se tomamos elementos da forma $g^{t} h^{s} e_{0}+g^{i} e(g) \hat{h}$, temos que tal soma é igual a $g^{t} e(g) \hat{h}+h^{s} e(h) \hat{g}+g^{i} e(g) \hat{h}=\left(g^{t}+g^{i}\right) e(g) \hat{h}+h^{s} e(h) \hat{g}$, donde recaímos na análise feita anteriormente. O mesmo vale para a soma $g^{t} h^{s} e_{0}+h^{j} e(h) \hat{g}$. Assim, L é fechado para a soma.

Além disso, dado $0 \neq r \in \mathcal{F}_{q}$, temos $r L=L$. De fato, $r g^{i} e(g) \hat{h}=g^{j} e(g) \hat{h}$, pois $\langle e(g)\rangle$ é essencial. Pela mesma razão $r h^{j} e(h) \hat{g}=h^{l} e(h) \hat{g}$ e, por fim, do mesmo argumento segue $r g^{t} h^{s} e_{0}=r\left(g^{t} e(g) \hat{h}+h^{s} e(h) \hat{g}\right)=g^{t_{1}} e(g) \hat{h}+h^{s_{1}} e(h) \hat{g}=g^{t_{1}} h^{s_{1}} e_{0}$.

Finalmente, é claro que $g L=L$ (bem como $h L=L)$ e, portanto, $L$ é ideal e o resultado segue.

Com isso temos o seguinte:

Corolário 2.13. O código $\mathcal{F}_{q}\left(C_{q^{m}-1} \times C_{q^{m}-1}\right) e_{0}$ é um código de dois pesos.

Baseados no Teorema 2.12, podemos calcular os pesos dos elementos de $\mathcal{F}_{q}\left(C_{q^{m}-1} \times C_{q^{m}-1}\right) e_{0}$.

Observe inicialmente que

$$
w(e(g) \hat{h})=w(e(g))\left(q^{m}-1\right) .
$$

Vamos então determinar o peso de e(g). Como e $(g)$ é idempotente essencial, $\operatorname{dim}_{\mathcal{F}_{q}} \mathcal{F}_{q} C_{q^{m}-1} e(g)=m$ e, como $\mathcal{F}_{q} C_{q^{m}-1} e(g)$ tem peso constante, pelo Teorema 2.7, $o$ peso de e $(g)$ é $\frac{q^{m-1}(q-1)\left(q^{m}-1\right)}{q^{m}-1}=q^{m-1}(q-1)$. O mesmo vale para $e(h)$, isto é, $w(e(h))=q^{m-1}(q-1)$. 
Chamemos de $\mathcal{C}=\mathcal{F}_{q}\left(C_{q^{m}-1} \times C_{q^{m}-1}\right) e_{0}$. Tal código tem dimensão $2 m$, donde a soma dos seus pesos é

$$
\sum_{\alpha \in \mathcal{C}} w(\alpha)=q^{2 m-1}(q-1)\left(q^{m}-1\right)^{2}
$$

Como $\mathcal{F}_{q} C_{q^{m-1}} e(g)$ e $\mathcal{F}_{q} C_{q^{m}-1} e(h)$ têm peso constante, a soma dos pesos em cada um desses conjuntos é $q^{m-1}(q-1)\left(q^{m}-1\right)$. Daí

$$
\begin{aligned}
w\left(g^{i} h^{j} e_{0}\right) & =\frac{q^{2 m-1}\left(q^{m}-1\right)^{2}(q-1)-2 q^{m-1}(q-1)\left(q^{m}-1\right)^{2}}{\left(q^{m}-1\right)^{2}} \\
& =q^{2 m-1}(q-1)-2 q^{m-1}(q-1) \\
& =\left(q^{2 m-1}-2 q^{m-1}\right)(q-1) .
\end{aligned}
$$

Assim, temos determinados os dois pesos do código $\mathcal{F}_{q}\left(C_{q^{m}-1} \times C_{q^{m}-1}\right) e_{0}$.

Exemplo 2.14. Para ilustrar o que discutimos acima, vamos trabalhar com o anel de grupo $\mathcal{F}_{3} C_{8}, C_{8}$ subgrupo cíclico com 8 elementos gerado por $g$. Comecemos encontrando um idempotente essencial, usando para isso o isomorfismo $\mathcal{F}_{3} C_{8}=\frac{\mathcal{F}_{3}(x)}{\left\langle x^{8}-1\right\rangle}$. Para tal, usaremos a tabela de polinômios irredutíveis encontrada em [10], a partir da página 553. Um dos polinômios fornecido pela tabela é

$$
\left(\begin{array}{lll}
1 & 1 & 2
\end{array}\right)=x^{2}+x+2=x^{2}+x-1=p(x) .
$$

A notação (1 112$)$, usada em [10], indica justamente as coordenadas do polinômio em $\mathcal{F}_{3}(x)$.

Considere o código isomorfo a $\frac{\left(\mathcal{F}_{3}(x)\right.}{\langle p(x)\rangle}$.

Queremos encontrar um polinômio e $(x)$ tal que ge seja raiz de $p(x)$ em $\mathcal{F}_{3} C_{8}$ e (em que e é a unidade), isto é, tal que $g^{2} e+g e-e=0$. Seja

$$
e(x)=a_{7} x^{7}+a_{6} x^{6}+a_{5} x^{5}+a_{4} x^{4}+a_{3} x^{3}+a_{2} x^{2}+a_{1} x+a_{0} .
$$

Podemos montar então um sistema de equações com coeficientes em $\mathcal{F}_{3}$ para encontrar cada um dos $a_{i}^{\prime} s$, usando para isso também o fato de que $e^{3}=e$. Para isso utilizamos a equação $g^{2} e+g e-e=0$ (encontrando para isso $g^{2} e$ e ge) e fazendo a igualdade de polinômios, além de tomar $a_{3}=a_{1}$ uma vez que $e^{3}=e\left(\right.$ pois $\left.e^{2}=e\right)$, obtemos o 
seguinte sistema de equações:

$$
\begin{aligned}
& a_{2}=-a_{1}+a_{0} \\
& a_{3}=-a_{2}+a_{1} \\
& a_{4}=-a_{3}+a_{2} \\
& a_{5}=-a_{4}+a_{3} \\
& a_{6}=-a_{5}+a_{4} \\
& a_{7}=-a_{6}+a_{5} \\
& a_{3}=a_{1}
\end{aligned}
$$

Resolvendo o sistema, encontramos que $a_{2}=0$, donde $a_{1}=a_{0}, a_{4}=-a_{3}, a_{5}=a_{4}$, $a_{6}=0$ e $a_{7}=a_{5}$. Tomando $a_{7}=-1$, encontramos como solução para o sistema

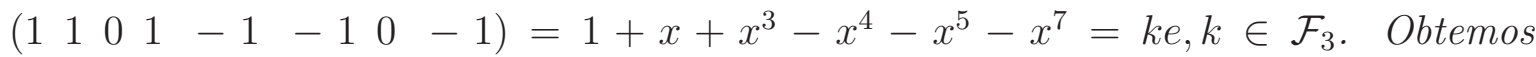
essas duas possibilidades de solução uma vez que utilizamos $e^{3}=e$. Como $e^{2}=e, e$ temos que $\left(1+x+x^{3}-x^{4}-x^{5}-x^{7}\right)^{2}=1+x+x^{3}-x^{4}-x^{5}-x^{7}$, concluímos que $k=1$ e, assim, $e=1+x+x^{3}-x^{4}-x^{5}-x^{7}$, em que g é tal que $C_{8}=\langle g\rangle$. Assim, e é essencial. De fato, os subgrupos próprios não triviais de $C_{8}$ são $\left\{1, g^{2}, g^{4}, g^{6}\right\}$ e $\left\{1, g^{4}\right\}$, e e $\times\left(1+g^{4}\right)=0=e \times\left(1+g^{2}+g^{4}+g^{6}\right)$, bem como $e \times \hat{g}=0$. Além disso, $\mathcal{F}_{3} C_{8}$ e tem peso constante, pois $\mathcal{F}_{3} C_{8}$ e é um código de comprimento 8, e, sendo $h(x)$ polinômio tal que $\mathcal{F}_{3} C_{8} e \simeq\langle h(x)\rangle$, como grau de $p(x)$ é dois, temos $\operatorname{gr}(h(x))=6$, e portanto pelo Teorema 1.54, a dimensão de $\mathcal{F}_{3} C_{8}$ e é $8-\operatorname{gr}(p(x))=8-6=2$, $e$ portanto $w(e)=6=\frac{3^{2-1} \times 2 \times 8}{3^{2}-1}$, donde temos satisfeita a condição 3 do Teorema 2.7. Conseguimos assim construir um código de dois pesos em $\mathcal{F}_{3}\left(C_{8} \times C_{8}\right)$, usando o idempotente essencial e construído acima, tomando o código $\mathcal{F}_{3}\left(C_{8} \times C_{8}\right)(e(g) \hat{h}+e(h) \hat{g})$.

Vejamos quais são os pesos de tal código. Efetuando a multiplicação, temos $w(e(g) \hat{h})=w\left(\left(1+g+g^{3}-g^{4}-g^{5}-g^{7}\right)\left(1+h+h^{2}+h^{3}+h^{4}+h^{5}+h^{6}+h^{7}\right)\right)=48$, uma vez que todos os termos da multiplicação terão suporte distintos. Além disso,

$$
\begin{gathered}
\left(1+g+g^{3}-g^{4}-g^{5}-g^{7}\right)\left(1+h+h^{2}+h^{3}+h^{4}+h^{5}+h^{6}+h^{7}\right)+ \\
+\left(1+g+g^{2}+g^{3}+g^{4}+g^{5}+g^{6}+g^{7}\right)\left(1+h+h^{3}-h^{4}-h^{5}-h^{7}\right)= \\
2+2 g+h^{6}+2 g^{3}+2 h-2 g^{4} h^{7}+2 g^{3} h+g^{3} h^{2}+2 g^{3} h^{3}+g^{3} h^{6} \\
+g^{2} h+g^{2} h^{3}-g^{2} h^{4}-g^{2} h^{5}-g^{2} h^{7}+2 g h+g h^{2}+2 g h^{3}+g h^{6}+g^{2}
\end{gathered}
$$




$$
\begin{gathered}
+2 h^{3}-g^{7} h^{2}-2 g^{7} h^{4}-2 g^{7} h^{5}-g^{7} h^{6}-2 g^{7} h^{7}+g^{6} h+g^{6} h^{3}-g^{6} h^{4}-g^{6} h^{5} \\
-g^{6} h^{7}-g^{5} h^{2}-2 g^{5} h^{4}-2 g^{5} h^{5}-g^{5} h^{6}-2 g^{5} h^{7}-g^{4} h^{2}-2 g^{4} h^{4}-2 g^{4} h^{5} \\
-g^{4} h^{6}+h^{2}+g^{6}
\end{gathered}
$$

donde $w(e(g) \hat{h}+e(h) \hat{g})=42$, que são justamente os pesos obtidos nos cálculos anteriores ao exemplo para o caso geral. 


\section{Capítulo 3}

\section{Códigos de peso constante em}

\section{outras classes de anéis de grupos}

Analisaremos aqui condições que garantam que um código tenha peso constante trabalhando agora com outras classes de anéis de grupos, usando novamente como ferramenta propriedades de anéis de grupo.

\subsection{Grupo Abeliano}

Começaremos trabalhando com o caso do anel de grupo $\mathcal{F}_{q} A$, em que $A$ é um grupo abeliano com $n$ elementos. Primeiramente, vamos assumir que $\operatorname{mdc}(n, q)=1$, isto é, que $\mathcal{F}_{q} A$ é um anel semissimples.

Teorema 3.1. Sejam $\mathcal{F}_{q}$ um corpo com $q$ elementos, $n$ um inteiro positivo com $\operatorname{mdc}(q, n)=1, A$ um grupo abeliano com $n$ elementos e $\mathcal{F}_{q} A$ o anel de grupo de $A$ sobre $\mathcal{F}_{q}$. Considere e um idempotente primitivo de $\mathcal{F}_{q} A$ e $\mathcal{C}=\mathcal{F}_{q} A$ e o respectivo código irredutivel. Seja $\operatorname{dim}_{\mathcal{F}_{q}} \mathcal{C}=k$. São equivalentes:

1. $\mathcal{C}$ tem peso constante;

2. Todo elemento não nulo de $\mathcal{C}$ tem peso $\frac{q^{k-1}(q-1) n}{q^{k}-1}$;

3. Existe um elemento de $\mathcal{C}$ cujo peso é $\frac{q^{k-1}(q-1) n}{q^{k}-1}$; 
4. $\left(\mathcal{F}_{q} A e\right)^{*}=\mathcal{F}_{q}^{*} A e$.

Prova: As implicações $(1 \Longrightarrow 2),(2 \Longrightarrow 3)$ e $(4 \Longrightarrow 1)$ são demonstradas de modo análogo ao Teorema 2.7. Vejamos então o caso $(3 \Longrightarrow 4)$. Caso o idempotente e seja essencial, a demonstração é similar a feita no Teorema 2.7, pois a argumentação só usa o fato de $\mathcal{F}_{q}$ Ae ser corpo finito, que resulta em $\left(\mathcal{F}_{q} A e\right)^{*}$ ser um grupo cíclico, o que ainda vale no caso em que A é abeliano. Analisemos por fim o caso em que e é não essencial. Observe que na demonstração do Teorema 2.7, para este caso, a argumentação foi baseada somente no fato de $\frac{C_{n}}{H_{e}}$ ser um grupo cíclico. Assim, se mostrarmos que $\frac{A}{H_{e}}$ é cíclico, com $H_{e}=\prod_{H \in \mathcal{H} e} H$, em que $\mathcal{H} e=\{H<A ; H e=e\}$, o resultado se demonstra de maneira análoga.

Considere então a função

$$
\begin{aligned}
\varphi: A & \rightarrow\left(\mathcal{F}_{q} A e\right)^{*} \\
a & \mapsto a e
\end{aligned}
$$

que é um homomorfismo de grupos. Calculemos $\operatorname{Ker}(\varphi)$. Seja a $\in \operatorname{Ker}(\varphi)$. Então $a e=e$, donde $a^{i} e=e$ e, assim, $\frac{1}{|\langle a\rangle|} \Sigma a^{i} e=e$, consequentemente, $\langle a\rangle \in \mathcal{H} e$, ou seja, $\langle a\rangle \subset H_{e}, \operatorname{logo} \operatorname{Ker}(\varphi) \subset H_{e} . S e h \in H_{e}, h e=h \hat{H}_{e} e=e$ e $\operatorname{ker}(\varphi)=H_{e}$. Disto, $\frac{A}{H_{e}} e^{\prime}$ isomorfo a um subgrupo de $\left(\mathcal{F}_{q} A e\right)^{*}$, que é cíclico, donde tal subgrupo é cíclico e temos $\operatorname{assim}(3 \Longrightarrow 4)$.

Trabalhemos agora com o caso em que A é um grupo abeliano com $n$ elementos, mas sem a hipótese de que $m d c(n, q)=1$, isto é, sem a hipótese que $\mathcal{F}_{q} A$ é semissimples. Seja $A=A_{p^{\prime}} \times A_{p}$, com $A_{p^{\prime}}$ o somando de $A$ cujos elementos têm ordem relativamente prima com $p$ e $A_{p}$ o p-subgrupo de Sylow de $A$. Então $\mathcal{F}_{q} A_{p^{\prime}}$ é um anel de grupo semissimples, digamos $\mathcal{F}_{q} A_{p^{\prime}}=\mathcal{F}_{q} A_{p^{\prime}} e_{1} \oplus \ldots \oplus \mathcal{F}_{q} A_{p^{\prime}} e_{t}$, com $e_{i}$ idempotentes primitivos e $\mathcal{F}_{q} A_{p^{\prime}} e_{i}=K_{i}$, com $K_{i}$ um corpo finito. Sob tais condições, concluímos que $\mathcal{F}_{q} A=\mathcal{F}_{q}\left(A_{p^{\prime}} \times A_{p}\right)=\left(\mathcal{F}_{q} A_{p^{\prime}}\right) A_{p}=\left(K_{1} \oplus \ldots \oplus K_{t}\right) A_{p}=K_{1} A_{p} \oplus \ldots \oplus K_{t} A_{p}$.

Vale o seguinte:

Lema 3.2. Com a notação acima, $K_{i} A_{p}$ é um anel local, para todo $1 \leq i \leq t$, cujo radical é o ideal de aumento $\operatorname{Ker}(\epsilon)$.

Prova: Seja $\operatorname{Ker}(\epsilon)=\left\{\alpha=\sum_{g \in A_{p}} \alpha_{g} g \in K_{i} A_{p} ; \Sigma \alpha_{g}=0\right\}$ o ideal de aumento. Seja $p^{r}$ o 
expoente de $A_{p}$. Então, se $\alpha=\sum_{g \in A_{p}} \alpha_{g} g$ pertence a $\operatorname{Ker}(\epsilon), \alpha^{p^{r}}=\left(\Sigma \alpha_{g} g\right)^{p^{r}}=\left(\Sigma \alpha_{g}\right)=$ 0. Seja $\beta$ um elemento de $K_{i} A_{p}$ não pertencente a seu ideal de aumento. Então $\epsilon(\beta)=k_{1} \neq 0$ e dai $\beta-k_{1} \in \operatorname{Ker}(\epsilon) . \operatorname{Logo} \beta=k_{1}+\left(\beta-k_{1}\right)=k_{1}\left(1+k_{1}^{-1}\left(\beta-k_{1}\right)\right)$. Vejamos que $1+k_{1}^{-1}\left(\beta-k_{1}\right)=1+\gamma$ é inversível, o que fará com que $\beta$ seja inversível. Como $\gamma \in \operatorname{Ker}(\epsilon), \gamma^{p^{r}}=0$. Daí, $(1+\gamma)\left(1-\gamma+\gamma^{2}-\ldots+(-1)^{p^{r-1}} \gamma^{p^{r-1}}\right)=1$, e $1+\gamma$ é inversivel. Como isso vale para qualquer elemento fora de $\operatorname{Ker}(\epsilon)$, segue que ele é maximal. Além disso é o conjunto dos não inversíveis de $K_{i} A$, e assim $K_{i} A$ é local com radical $\operatorname{Ker}(\epsilon)$.

Seja $\tilde{A}_{p}=\sum_{a \in A_{p}} a$ e $\left\langle\tilde{A}_{p}\right\rangle=K_{i} A_{p} \tilde{A}_{p}$ o ideal de $K_{i} A_{p}$ gerado por $\tilde{A}_{p}$. Vale então:

Lema 3.3. Com a notação introduzida acima, $\left\langle\tilde{A}_{p}\right\rangle$ é o único ideal irredutível de $K_{i} A_{p}$.

Prova: De fato, $\left\langle\tilde{A}_{p}\right\rangle$ é minimal, pois se $0 \neq J \subseteq\left\langle\tilde{A}_{p}\right\rangle$, então, dado $x \in J, x=k_{i} \tilde{A}_{p}$, com $k_{i} \in K_{i}$. Daí, como $J$ é ideal, $\tilde{A}_{p}=k_{i}^{-1} k_{i} \tilde{A}_{p} \in J$, e $J=\left\langle\tilde{A}_{p}\right\rangle$. Disto $\left\langle\tilde{A}_{p}\right\rangle$ é irredutível. Vejamos que ele é o único.

Suponha $I$ um ideal irredutivel de $K_{i} A_{p}$. Então $\operatorname{Ker}(\epsilon) I \subseteq I=0$ ou $I$. Se $M I=I$, pelo Lema de Nakayama, $I=0$. Disto $M I=0$. Dado qualquer $a \in A_{p}, 1-a \in$ $\operatorname{Ker}(\epsilon)$. Logo, para $\alpha \in I, \alpha(1-a)=0$. Como $\alpha=\sum_{g \in A_{p}} \alpha_{g} g$ e $(1-a) \alpha=0$, temos $\Sigma \alpha_{g}(1-a)=0$, donde todas as coordenadas de $\Sigma \alpha_{g}(1-a)$ são nulas, em particular a coordenada que acompanha $a \in G$, isto é, $\alpha_{1}-\alpha a=0$ e, assim, $\alpha_{a}=\alpha_{1}$. E isto vale para todo $a \in A_{p}$, donde $\alpha=k_{i} \tilde{A}_{p}$ e, portanto, $I=\left\langle\tilde{A}_{p}\right\rangle$.

Como os ideais irredutíveis de $\mathcal{F}_{q} A$ são os ideais irredutíveis de $K_{i} A_{p}$, uma vez que $\mathcal{F}_{q} A=K_{1} A_{p} \oplus \cdots \oplus K_{t} A_{p}$, temos como consequência

Corolário 3.4. Os ideais irredutíveis de $\mathcal{F}_{q} A$ são da forma $\mathcal{F}_{q} A_{p^{\prime}} e_{i} \tilde{A}_{p}$, para todo $1 \leq i \leq t$.

Temos ainda o seguinte resultado:

Lema 3.5. Seja $\mathcal{F}_{q}$ um corpo finito com $q$ elementos e $A$ um grupo abeliano com $n$ elementos. Se um código de $\mathcal{F}_{q}$ A tem peso constante, então ele é irredutível. 
Prova: É a mesma do caso em que o grupo é um grupo cíclico, veja Lema 2.6.

Assim, para que um código de $\mathcal{F}_{q}$ A seja de peso constante, ele deve ser irredutivel, e pelo Lema 3.3, o código deve ser da forma $\mathcal{F}_{q} A_{p^{\prime}} e_{i} \tilde{A}_{p}$. Como um elemento de tal conjunto é da forma $\alpha=\alpha_{1} \tilde{A}_{p}$ (e para cada $g \neq h \in A_{p}$, supp $\alpha_{1} g$ e suppo $\alpha_{1}$ são conjuntos disjuntos), com $\alpha_{1} \in \mathcal{F}_{q} A_{p^{\prime}} e_{i}$, seu peso é $\omega\left(\alpha_{1}\right)\left|A_{p}\right|$, ou seja, a análise do peso dos elementos do código se restringe a análise do peso dos elementos de $\mathcal{F}_{q} A_{p^{\prime}} e_{i}$. Disto, pelo já visto no caso anterior, vale:

Teorema 3.6. Sejam $\mathcal{F}_{q}$ um corpo finito com $q$ elementos, $n$ um inteiro positivo com $m d c(q, n) \neq 1, A$ um grupo abeliano com $n$ elementos e $\mathcal{F}_{q} A$ o anel de grupo de $A$ sobre $\mathcal{F}_{q}$. Considere e um idempotente primitivo de $\mathcal{F}_{q} A$ e $\mathcal{C}=\mathcal{F}_{q} A$ e $=\mathcal{F}_{q} A_{p^{\prime}}$ e $\tilde{A}_{p}$ o respectivo código irredutivel. Seja $\operatorname{dim}_{\mathcal{F}_{q}} \mathcal{C}=k$. Então $\mathcal{C}$ tem peso constante se, $e$ somente se, dados todos os elementos $\alpha=\alpha_{1} \tilde{A}_{p}$, com $\alpha_{1} \in \mathcal{F}_{q} A_{p^{\prime}}$ e, têm o mesmo peso, isto é, se, e somente se, $\mathcal{F}_{q} A_{p^{\prime}} e_{i}$ tem peso constante.

Observe que, com tal teorema, temos em particular uma caracterização para os códigos de $\mathcal{F}_{q} C_{n}$, com $C_{n}$ um grupo cíclico, para o caso em que $\mathcal{F}_{q} C_{n}$ não é semissimples.

\subsection{Domínio de Integridade}

Seja $R$ um domínio de integridade infinito e A um grupo abeliano finito. Nessas condições, tomando o anel de grupos RA, embora não possamos falar nos ideais de RA como códigos, ainda assim podemos olhar o peso de Hamming dos elementos destes ideais. Para este caso, temos o seguinte:

Teorema 3.7. Sejam $R$ um dominio de integridade infinito e A um grupo abeliano finito. Considere o anel de grupo RA e seja I um ideal de RA. Então $w(I)=|A|$ se, e somente se, I tem peso constante.

Prova: Seja I um ideal de $R A$ e suponha $w(I) \neq|A|$. Então existe $\alpha \in I$ com $w(\alpha) \neq|A|$. Assim, se $\alpha=\sum_{h \in A} \alpha_{h} h$, existe $g \in A$ tal que $\alpha_{g}=0$. Suponha que $\alpha_{1} \neq 0$ (se $\alpha_{1}=0$, basta multiplicar $\alpha$ por $\left(g^{\prime}\right)^{-1}$, com $\alpha_{g^{\prime}} \neq 0$ e teremos o desejado ). Como $A$ 
é finito, existem finitos $h \in A$ tais que o coeficiente $\alpha_{h} \neq 0$. Como $R$ é um domínio de integridade, existe $k \in R$ tal que $\alpha_{h} \neq k \alpha_{h g^{-1}}$, para todo $h$ com $\alpha_{h} \neq 0$. Em particular como $\alpha_{1} \neq 0$, temos $k \alpha_{1} \neq 0$. Com esse $k$, tome o elemento $\alpha-k g \alpha$. Tal elemento está em I, uma vez que I é ideal. Para todo $h \in A$, o coeficiente de $h$ em $\alpha-k g \alpha$ será $\alpha_{h}-k \alpha_{h g^{-1}}$. Pela escolha de $k$, os coeficientes $\alpha_{h}$ de $\alpha$ que são não nulos continuam sendo coeficientes diferentes de 0 em $\alpha-k g \alpha$ (pois o coeficiente de $h \in A$ em $\alpha-k g \alpha$ é $\left.\alpha_{h}-k \alpha_{h g^{-1}}\right)$. Além disso, o coeficiente de g em $\alpha-k g \alpha$ será $\alpha_{g}-k \alpha_{g} g^{-1}=k \alpha_{1} \neq 0$. Assim, $w(\alpha)<w(\alpha-k g \alpha)$, donde I não tem peso constante. Portanto, para I ter peso constante é necessário que $w(I)=|A|$.

Por outro lado, se $w(I)=|A|$, então, como o maior peso possível é $|A|$, segue imediatamente que I tem peso constante.

Exemplo 3.8. Seja $K$ um corpo infinito e A um grupo abeliano finito. Analisemos os ideais de peso constante de $K A$ :

Pelo teorema acima, um ideal I de KA tem peso constante se, e somente se, $w(I)=|A|$. Seja I um ideal de $K A$.

Se $\operatorname{dim}_{k}(I)=1$, então existe $\alpha \in I$ tal que $I=\{k \alpha \mid k \in K\}$. Neste caso, todos os elementos de I têm o mesmo peso de $\alpha$, ou seja, I tem peso constante, donde $w(\alpha)=|A|$.

Agora, seja I um ideal não nulo de KA e suponha que todos os elementos não nulos de I tenham o mesmo peso. Vejamos que neste caso $I$ deve ter dimensão 1 sobre $K$. Seja $\alpha=\sum_{g \in A} \alpha_{g} g$ um elemento não nulo de $I$, e suponha que exista $\beta=\sum_{g \in A} \beta_{g} g \in I$ tal que $\beta \neq r \alpha$, para todo $r \in K$. Como $w(I)=|A|, \alpha_{g} \neq 0 \neq \beta_{g}$, para todo $g \in A$. Em particular, $\alpha_{1} \neq 0 \neq \beta_{1}$. Tome $k=\alpha_{1}\left(\beta_{1}\right)^{-1} \neq 0$. Então $k \beta=\alpha_{1}+\sum_{g \in A, g \neq 1} k \beta_{g} g$. Como $\beta \neq r \alpha$, para todo $r \in K, \beta \neq k^{-1} \alpha$, isto é, $k \beta \neq \alpha$. Considere $\gamma=k \beta-\alpha \neq$ 0. Temos $\gamma \in I$. Mas $\gamma_{1}=k \beta_{1}-\alpha_{1}=\alpha_{1}\left(\beta_{1}\right)^{-1} \beta_{1}-\alpha_{1}=\alpha_{1}-\alpha_{1}=0$, donde $w(\gamma)<|A|$, contradizendo o fato de que todos os elementos de I têm peso $|A|$. Disto $I=\{r \alpha \mid r \in K\}$, isto é, $\operatorname{dim}_{k}(I)=1$. 


\subsection{Anel de Cadeia}

Seja $R$ um anel de cadeia finito, com ideal maximal $\langle p\rangle$ e com cadeia de ideais $0 \subseteq\left\langle p^{r-1}\right\rangle \subseteq \ldots \subseteq\left\langle p^{2}\right\rangle \subseteq\langle p\rangle$, em que $r$ é o índice de nilpotência de $p$. Seja $\frac{R}{\langle p\rangle}=\bar{R}=\mathbb{F}_{q^{s}}$, com q potência de um número primo.

Considere $G=\langle g\rangle$ um grupo cíclico com $n$ elementos e o anel de grupo RG. Suponha que $m d c(q, n)=1$. Então $\bar{R} G$ é semissimples, donde existem $e_{1}, e_{2}, \ldots, e_{k}$ idempotentes primitivos ortogonais em $\bar{R} G$ tais que

$$
\bar{R} G=\bar{R} G e_{1} \oplus \ldots \oplus \bar{R} G e_{k}
$$

e, pelo Teorema 1.42, existem $e_{1}^{\prime}, \ldots, e_{k}^{\prime}$ idempotentes primitivos ortogonais em $R G$, com $\bar{e}_{i}^{\prime}=e_{i}$ e tais que

$$
R G=R G e_{1}^{\prime} \oplus \ldots \oplus R G e_{k}^{\prime} .
$$

Vamos agora analisar os códigos de peso constante em $R G e_{i}^{\prime}$. Para isso, vejamos inicialmente o seguinte resultado:

Lema 3.9. A função:

$$
\begin{aligned}
\Psi^{\prime}:(\bar{R},+) & \rightarrow\left(\left\langle p^{r-1}\right\rangle,+\right) \\
\bar{x} & \mapsto p^{r-1} x
\end{aligned}
$$

com $\left\langle p^{r-1}\right\rangle$ visto como ideal de $R$, é um isomorfismo de grupos que dá origem, para cada $e_{i}$ idempotente em $\bar{R} G$, à bijeção

$$
\begin{aligned}
\Psi: \bar{R} G e_{i} & \rightarrow\left\langle p^{r-1} e_{i}^{\prime}\right\rangle \\
\sum_{g \in G} \bar{\alpha}_{g} g e_{i} & \mapsto p^{r-1} \sum_{g \in G} \alpha_{g} g e_{i}^{\prime}
\end{aligned}
$$

com $\left\langle p^{r-1} e_{i}^{\prime}\right\rangle$ visto como ideal de RG. Tal bijeção preserva soma e peso.

Prova: Note que $\Psi^{\prime}$ está bem definida, pois dados $x, y \in R$ com $\bar{x}=\bar{y}$, então $x-y=p a, a \in R$. Assim, $p^{r-1}(x-y)=p^{r-1} p a=0$, donde $p^{r-1} x=p^{r-1} y$, isto é, $\Psi^{\prime}(\bar{x})=\Psi^{\prime}(\bar{y})$. Além disso, dados $\bar{x}, \bar{y} \in \bar{R}, \Psi^{\prime}(\bar{x}+\bar{y})=\Psi^{\prime}(\overline{x+y})=p^{r-1}(x+y)=$ $\Psi^{\prime}(\bar{x})+\Psi^{\prime}(\bar{y})$. Também, se $\Psi^{\prime}(\bar{x})=0$, então $p^{r-1} x=0$, donde $x=p a, a \in R$, logo $\bar{x}=0$. Por fim, dado $p^{r-1} x \in\left\langle p^{r-1}\right\rangle$, basta tomar $\bar{x} \in \bar{R}$ e temos $\Psi^{\prime}(\bar{x})=p^{r-1} x$. Portanto $\Psi^{\prime}$ é isomorfismo de grupos. 
Como $\Psi^{\prime}$ é isomorfismo e dele se origina $\Psi$, é imediato que $\Psi$ é bijeção e preserva soma. Além disso,

$$
\begin{aligned}
w\left(p^{r-1} x\right) & =\left\{x_{g} \in \operatorname{supp}(x) \mid x_{g} \text { é unidade }\right\} \\
& =\left\{\bar{x}_{g} \in \operatorname{supp}(\bar{x}) \mid \bar{x}_{g} \neq 0\right\} \\
& =w(\bar{x})
\end{aligned}
$$

e, portanto, $\Psi$ preserva peso.

Observe que com tal resultado podemos concluir que se $\left\langle p^{r-1} e_{i}^{\prime}\right\rangle$ tem peso constante em $R G, e_{i}$ deve gerar um ideal de peso constante em $\bar{R} G$, isto é, se $e_{i}$ gera um ideal que não tem peso constante, então o ideal gerado por $e_{i}^{\prime}$ não pode ter peso constante, uma vez que $\left\langle p^{r-1} e_{i}^{\prime}\right\rangle$ não terá peso constante e $\left\langle p^{r-1} e_{i}^{\prime}\right\rangle \subseteq\left\langle e_{i}^{\prime}\right\rangle$. Mais ainda, concluímos que um ideal de $R G e_{i}^{\prime}$ tem peso constante se, e somente se, $\bar{R} G e_{i}$ tem peso constante.

Lema 3.10. Se $I^{\prime}$ é um ideal de $R G$ de peso constante, então $I^{\prime}$ é indecomponível.

Prova: Tome $I^{\prime}$ ideal de $R G$ decomponível, digamos $I^{\prime}=I_{1} \oplus I_{2}$. Então $I_{1}$ contém um minimal $\left\langle p^{r-1} e_{1}^{\prime}\right\rangle$, bem como $I_{2}$ contém um minimal $\left\langle p^{r-1} e_{2}^{\prime}\right\rangle$, com $e_{1}^{\prime} \neq e_{2}^{\prime}$. Então $\left\langle p^{r-1}\left(e_{1}^{\prime}+e_{2}^{\prime}\right)\right\rangle \subseteq\left\langle p^{r-1} e_{1}^{\prime}\right\rangle \oplus\left\langle p^{r-1} e_{2}^{\prime}\right\rangle \subseteq I$. Como um subideal de um ideal de peso constante tem peso constante, o ideal $\left\langle p^{r-1}\left(e_{1}^{\prime}+e_{2}^{\prime}\right)\right\rangle$ tem peso constante, donde, pelo Lema anterior, sendo $\overline{e_{1}^{\prime}}=e_{1}, \overline{e_{2}^{\prime}}=e_{2},\left\langle\left(e_{1}+e_{2}\right)\right\rangle$ deve ter peso constante em $\bar{R} G$. Mas isto não ocorre, uma vez que se um código em $\bar{R} G$ tem peso constante então ele deve ser irredutivel de acordo com o Lema 2.6. Portanto, para um ideal ter peso constante em $R G$ ele precisa ser indecomponível.

De acordo com o Teorema 1.39, sabemos que os ideais de $R G e_{i}^{\prime}$ são da forma $\left\langle p^{j} e_{i}^{\prime}\right\rangle$ para $0 \leq j \leq r-1$ e $1 \leq i \leq n$. Assim, dado $I^{\prime}$ um ideal de $R G e_{i}^{\prime}$, ele deve ser igual a algum $\operatorname{dos}\left\langle p^{j} e_{i}^{\prime}\right\rangle$.

Podemos então dizer o seguinte:

Teorema 3.11. Sejam $R$ um anel de cadeia finito com ideal maximal $\langle p\rangle, p$ com índice de nilpotência $r, \frac{R}{\langle p\rangle}=\bar{R} \simeq \mathcal{F}_{q}, \mathcal{F}_{q}$ corpo finito com $q$ elementos, e $G$ um grupo cíclico com $n$ elementos tal que $\operatorname{mdc}(q, n)=1$. Considere $e_{i}$ idempotente primitivo de $\bar{R} G$ que gera um código de peso constante, $e_{i}^{\prime}$ idempotente primitivo de $R G$, com $\bar{e}_{i}^{\prime}=e_{i}$, 
e seja $I^{\prime}$ um ideal de $R G e_{i}^{\prime}$. Se $w\left(e_{i}\right) \neq|G|, I^{\prime}$ tem peso constante se, e somente se, $I^{\prime}=\left\langle p^{r-1} e_{i}^{\prime}\right\rangle$

Prova: Seja $I=\bar{R} G e_{i}$ código não nulo de peso constante e assuma $w(I) \neq|G|$. Então, pelo Lema 3.9, temos que $\left\langle p^{r-1} e_{i}^{\prime}\right\rangle$ tem peso constante. Vejamos que neste caso, para um código $I^{\prime}$ em $R G e_{i}^{\prime}$ ter peso constante, ele precisa ser da forma $\left\langle p^{r-1} e_{i}^{\prime}\right\rangle$.

Suponha, por absurdo, que exista $I^{\prime}=\left\langle p^{j} e_{i}^{\prime}\right\rangle$, com $j<r-1$ de peso constante. Como $w(I) \neq|G|$, existe $g_{i} \in G$, para $e_{i}=\sum_{g \in G} a_{g} g$, com $a_{g_{i}}=0$. Assim $e_{i}^{\prime}$ tem coordenadas que são unidades e coordenadas que ou são nulas ou são múltiplas de p. Se todas as coordenadas de $p^{j} e_{i}^{\prime}$ são não nulas, então é porque as coordenadas de $e_{i}^{\prime}$ também são não nulas, e pelo fato de $e_{i}$ ter coordenadas nulas segue que $e_{i}^{\prime}$ tem coordenadas em $\langle p\rangle$. Disto $\left\langle p^{j} e_{i}^{\prime}\right\rangle$ não poderá ter peso constante, pois nesse caso tal coordenada estará em $\left\langle p^{j+1}\right\rangle$, mas há coordenadas de $p^{j} e_{i}^{\prime}$ que estão em $\left\langle p^{j}\right\rangle \backslash\left\langle p^{j+1}\right\rangle$ (pois há coordenadas de $e_{i}^{\prime}$ que são unidades) e, assim, multiplicando $p^{j} e_{i}^{\prime}$ por $p^{r-1-j}$, obteremos $w\left(p^{j} e_{i}^{\prime}\right)>w\left(p^{r-1-j} p^{j} e_{i}^{\prime}\right)$. Assim, podemos assumir que $p^{j} e_{i}^{\prime}$ tem alguma coordenada nula.

Tomemos então $\alpha \in\left\langle p^{j} e_{i}^{\prime}\right\rangle, \alpha=\sum_{g \in G} \alpha_{g} g$, tal que algumas das coordenadas $\alpha_{g}^{\prime \prime}$ de $\alpha$ é nulo. Como $I^{\prime}=\left\langle p^{j} e_{i}^{\prime}\right\rangle$, podemos tomar este $\alpha$ tal que exista $g^{\prime} \in G$ com $\alpha_{g}^{\prime} \in\left\langle p^{j}\right\rangle \backslash\left\langle p^{j+1}\right\rangle$. Temos dois casos a considerar:

Caso 1: todas as coordenadas não nulas de $\alpha$ estão em $\left\langle p^{j}\right\rangle \backslash\left\langle p^{j+1}\right\rangle$ :

Neste caso, multiplicamos $\alpha$ por $p\left(g^{\prime}\right)^{-1} g^{\prime \prime}$. Neste caso, tomando $\alpha+p\left(g^{\prime}\right)^{-1} g^{\prime \prime} \alpha$, como as coordenadas de $\alpha$ estão em $\left\langle p^{j}\right\rangle \backslash\left\langle p^{j+1}\right\rangle$ e as coordenadas de $p\left(g^{\prime}\right)^{-1} g^{\prime \prime} \alpha$ estão em $\left\langle p^{j+1}\right\rangle$, as coordenadas não nulas de $\alpha$ continuam não nulas em $\alpha+p\left(g^{\prime}\right)^{-1} g^{\prime \prime} \alpha$. Além disso, a coordenada de $g^{\prime \prime}$, que em $\alpha$ é nula, em $\alpha+p\left(g^{\prime}\right)^{-1} g^{\prime \prime} \alpha$ é diferente de 0 (pois é igual a $a_{g^{\prime}} p$, e $a_{g} \in\left\langle p^{j}\right\rangle \backslash\left\langle p^{j+1}\right\rangle$, com $\left.j<r-1\right)$. Disto $w\left(\alpha+p\left(g^{\prime}\right)^{-1} g^{\prime \prime} \alpha\right)>w(\alpha)$, uma contradição.

Caso 2: alguma das coordenadas não nulas de $\alpha$ estando em $\left\langle p^{j+1}\right\rangle$ :

Assim, multiplicando $\alpha$ por $p^{r-1-j}$, teremos pela mesma argumentação do caso anterior $w(\alpha)>w\left(p^{r-1-j} \alpha\right)$, uma contradição. Portanto, para ter peso constante, $I^{\prime}=\left\langle p^{r-1} e_{i}^{\prime}\right\rangle$. 
Reciprocamente, seja $I^{\prime}$ ideal em $R G e_{i}^{\prime}$ e suponha que $\left\langle e_{i}\right\rangle$ não tem peso constante. Então existem $x, y \in\left\langle e_{i}\right\rangle$ com $|\operatorname{supp}(x)| \neq|\operatorname{supp}(y)|$, digamos $|\operatorname{supp}(x)|>|\operatorname{supp}(y)|$ (o outro caso é análogo). Então, dados levantamentos $x^{\prime}=\sum_{g \in G} \alpha_{g} g$ e $y^{\prime}=\sum_{g \in G} \beta_{g} g d e$ $x$ e $y$, respectivamente, como existe ao menos um $g^{\prime} \in G$ a mais em $y$, comparado a $x$, tal que a coordenada que acompanha $g^{\prime}$ é nula, para tal $g^{\prime}$ temos $\beta_{g^{\prime}} \in\langle p\rangle$, isto é, $\beta_{g^{\prime}}=p \gamma_{g^{\prime}}$. Daí, ou $\gamma_{g^{\prime}}=0$, para todo $g^{\prime}$ com esta característica e, neste caso, $w\left(x^{\prime}\right)>w\left(y^{\prime}\right)$, ou algum $\gamma_{g^{\prime}} \neq 0$, e daí, como $p^{r-1} y^{\prime} \in I^{\prime}$, teremos $w\left(x^{\prime}\right)>w\left(p^{r-1} y^{\prime}\right)$. Em qualquer dos casos concluímos que $I^{\prime}$ não tem peso constante, donde para $I^{\prime}$ ter peso constante I deve ter peso constante.

Para o caso em que $w\left(e_{i}\right)=|G|$, vale o seguinte.

Teorema 3.12. Sejam $R$ um anel de cadeia finito com ideal maximal $\langle p\rangle, p$ com índice de nilpotência $r, \frac{R}{\langle p\rangle}=\bar{R} \simeq \mathcal{F}_{q}, \mathcal{F}_{q}$ um corpo finito com $q$ elementos, e $G$ um grupo cíclico com $n$ elementos tal que $\operatorname{mdc}(q, n)=1$. Considere $e_{i}$ idempotente primitivo de $\bar{R} G$ que gera um ideal de peso constante e $e_{i}^{\prime}$ idempotente primitivo de $R G$, com $\bar{e}_{i}^{\prime}=e_{i}$. Suponha $w\left(e_{i}\right)=|G|$. Então $R G e_{i}^{\prime}$, bem como todos os seus subideais, tem peso constante.

Prova: Suponha $w\left(e_{i}\right)=|G|$. Então, para $e_{i}=\sum_{g \in G} a_{g} g, a_{g} \neq 0$, para todo $g$, e portanto, para qualquer levantamento de $e_{i}$ e, em particular, para $e_{i}^{\prime}$, suas coordenadas são todas unidades. Como $w\left(e_{i}\right)=|G|$, temos que $\left\langle e_{i}\right\rangle$ tem dimensão 1. De fato, como $\left\langle e_{i}\right\rangle$ tem peso constante, $w\left(e_{i}\right)=\frac{q^{k-1} n(q-1)}{q^{k}-1}=n$, então $\frac{q^{k-1}(q-1)}{q^{k}-1}=1$, isto é, $q^{k}-q^{k-1}=q^{k}-1$ donde $q^{k-1}=1$ e consequentemente $k=1$. Neste caso, $\left|\left\langle e_{i}\right\rangle\right|=q$, donde $\left|\left\langle p^{r-1} e_{i}^{\prime}\right\rangle\right|=q$ e $\left|\left\langle e_{i}^{\prime}\right\rangle\right|=q^{r}$. Assim, os elementos de $\left\langle e_{i}^{\prime}\right\rangle$ são da forma $k e_{i}^{\prime}, k \in R$, pois dados $k_{1}, k_{2} \in R, k_{1} e_{i}^{\prime} \neq k_{2} e_{i}^{\prime}$, uma vez que as coordenadas de $e_{i}^{\prime}$ são unidades. Disto todo elemento de $\left\langle e_{i}^{\prime}\right\rangle$ tem peso $|G|$ e, portanto, $\left\langle e_{i}^{\prime}\right\rangle$ tem peso constante, donde $\left\langle p^{j} e_{i}^{\prime}\right\rangle$ tem peso constante, para $0 \leq j \leq r-1$.

Para o nosso último exemplo, precisaremos da seguinte definição:

Definição 3.13. Dado A um grupo abeliano finito, $g$ um elemento de $A$ e q um número primo, a classe $q$-ciclotômica de $g$ é o conjunto

$$
S_{g}=\left\{g^{q^{j}} \mid 0 \leq j \leq t_{g}-1\right\},
$$


em que $t_{g}$ é o menor inteiro positivo tal que $q^{t_{g}} \equiv 1(\operatorname{modo}(g))$.

Em [6] encontramos o seguinte resultado, que pode ser visto como uma generalização de um resultado de Berman [1] e Witt [20]:

Teorema 3.14. Seja $F$ um corpo finito, com $|F|=q$, e seja $A$ um grupo abeliano finito com $m d c(q,|A|)=1$. Então o número de componentes simples de FA é igual ao número de classes q-ciclotômicas de A.

Exemplo 3.15. Vamos analisar quais são os ideais de peso constante em $\mathbb{Z}_{8} C_{7}$.

Observe que $\mathbb{Z}_{8}$ é um anel de cadeia com cadeia de ideais

$$
\mathbb{Z}_{8} \supseteq\langle 2\rangle \supseteq\langle 4\rangle \supseteq(0)
$$

Então, pelos resultados anteriores, para determinar os ideais de peso constante de $\mathbb{Z}_{8} C_{7}$, basta definirmos os de peso constante em $\overline{\mathbb{Z}}_{8} C_{7}=\mathcal{F}_{2} C_{7}$. Para isso, estabelecemos inicialmente os idempotentes primitivos ortogonais de $\mathcal{F}_{2} C_{7}$. Com um cálculo similar ao feito no Exemplo 2.14, encontramos os idempotentes $e_{1}=\hat{g}, e_{2}=1+g+g^{2}+g^{4}$, e $e_{3}=1+g^{3}+g^{5}+g^{6}$. Um cálculo direto mostra que tais idempotentes são dois a dois ortogonais, com soma 1. Além disso, as classes 2-ciclotômicas de $C_{7}$ são $\{1\}$, $\left\{g^{2}, g^{4}, g^{6}\right\}$ e $\left\{g^{3}, g^{5}, g^{6}\right\}$, isto é, são 3 classes 2-ciclotômicas, donde são 3 as componentes simples de $\mathcal{F}_{2} C_{7}$ e, portanto, tais idempotentes são primitivos. Logo $\left\{e_{1}, e_{2}, e_{3}\right\}$ é um conjunto completo de idempotentes ortogonais primitivos. Ainda, $\operatorname{dim}(\langle\hat{g}\rangle)=1$, e $w(\hat{g})=7=\frac{2^{1-1}(2-1) 7}{2^{1}-1}$, bem como $\operatorname{dim}\left(\left\langle e_{1}\right\rangle\right)=\operatorname{dim}\left(\left\langle e_{1}\right\rangle\right)=3=\frac{2^{3-1}(2-1) 7}{2^{3}-1}$ (tais dimensões obtidas usando o grau do polinômio que gera cada um desses idempotentes e com o Teorema 1.54, tal como foi feito no Exemplo 2.14), donde pelo Teorema 2.7 tais idempotentes geram códigos de peso constante.

Na demonstração do Teorema 1.28, é feita a construção do idempotente em $R$ dado um idempotente em $\bar{R}$. Usando tal construção, concluímos que os idempotentes associados a $e_{1}, e_{2}$ e e $e_{3}$ são, respectivamente, $e_{1}^{\prime}=7 \hat{g}, e_{2}^{\prime}=5+3 g+3 g^{2}+6 g^{3}+3 g^{4}+$ $6 g^{5}+6 g^{6}, e_{3}^{\prime}=5+6 g+6 g^{2}+3 g^{3}+6 g^{4}+3 g^{5}+3 g^{6}$. Assim, os códigos de peso constante de $\mathbb{Z}_{8} C_{7}$ devem ser ideais de $\left\langle e_{1}^{\prime}\right\rangle,\left\langle e_{2}^{\prime}\right\rangle$ e $\left\langle e_{3}^{\prime}\right\rangle$. É fácil ver que $\left\langle e_{1}^{\prime}\right\rangle$ tem peso constante, donde seus ideais também têm peso constante. Já para $\left\langle e_{2}^{\prime}\right\rangle$, note que $w\left(4 e_{2}^{\prime}\right)<w\left(e_{2}^{\prime}\right)$, donde $\left\langle e_{2}^{\prime}\right\rangle$ bem como $\left\langle 2 e_{2}^{\prime}\right\rangle$ não têm peso constante. Já $\left\langle 4 e_{2}^{\prime}\right\rangle$ tem peso constante pelo Lema 3.7, uma vez que $\left\langle e_{2}\right\rangle$ tem peso constante. O mesmo vale para a análise de $\left\langle e_{3}^{\prime}\right\rangle$ 
e seus ideais. Logo, como nos teoremas acima, os ideais de peso constante de $\mathbb{Z}_{8} C_{7}$ são $\left\langle e_{1}^{\prime}\right\rangle$ (e seus ideais), $\left\langle 4 e_{2}^{\prime}\right\rangle$ e $\left\langle 4 e_{3}^{\prime}\right\rangle$. 


\section{Capítulo 4}

\section{Conclusão}

Nesta tese trabalhamos com códigos sobre os seguintes anéis de grupo :

- $\mathcal{F}_{q} A$, com $\mathcal{F}_{q}$ um corpo finito com q elementos e $A$ um grupo abeliano finito;

- $R C_{n}$, com $R$ um anel de cadeia finito e $C_{n}$ um grupo cíclico com $n$ elementos tal que $\operatorname{mdc}(n, q)=1$.

As contribuições deste trabalho foram dar condições que garantissem que um código tenha peso constante nessas classes de anéis de grupo, além da construção de códigos de dois pesos usando os códigos de peso constante em $\mathcal{F}_{q} C_{q}^{n-1}$.

Como perspectivas futuras, pretendemos analisar códigos de peso constante sobre $R A$, em que $R$ é um anel local finito e $A$ é um grupo abeliano finito. Também queremos trabalhar com códigos de exatamente dois pesos. 


\section{Referências Bibliográficas}

[1] S.D.Berman, The number of irreducible representations of a finite group over an arbitrary field.(Russian) Dokl. Akad. Nauk. 106: 767 - 769, (1956).

[2] G. Chalom, R. A. Ferraz and C. Polcino Milies, Essential Idempotents in Abelain Group Algebras (submetido).

[3] Y. M. Chee and S. Ling Constructions for q-ary constant-weight codes, IEEE Transactions on Information Theory, vol 53, no 1: 135 - 146, (2007).

[4] H. Q. Dinh, S. R. López-Permouth, Cyclic and negacyclic codes over finite chain rings, IEEE Transactions on Information Theory, Vol 50: 1728 - 1744, (2004).

[5] T. Etzion, A. Vardy, A new construction for constant weight codes, Arxive - 3 mar 2014 .

[6] R. A. Ferraz and C.Polcino Milies, Idempotents in group algebras and minimal abelian codes, Finite Fields and their Applications 13, 382-393 (2007).

[7] R.A.Ferraz, C.Polcino Milies and M. Guerreiro, G-equivalence in group algebras and minimal abelian codes, IEEE Transactions on Information Theory, vol 60, no1: 252 - 260, (2014).

[8] J.B, Fraleigh A First Course in Abstract Algebra, Fifth Edition, Addison-Wesley, (1997).

[9] N. Jacobson, Basic Algebra II, W.H. Freeman and Company, San Francisco, (1980). 
[10] R. Lidl and H. Niederreiter Finite Fields, Cambridge University Press, Second Edition, (1997).

[11] B.R. MacDonald, Finite Rings with Identity, A series of monographs and Textbooks, New York, (1974).

[12] H. Matsumura, Commutative Algebra, Second Edition, The Benjamin Cummings Publishing Company, Massachusettis, (1980).

[13] C. Polcino Milies, Breve Introdução à Teoria dos Códigos Corretores de Erros, Departamento de Matemática, UFMS, (2009).

[14] C.Polcino Milies and S.K. Sehgal, An Introduction to Group Rings, Kluwer Academic Publishers, (2002).

[15] S. Roman, Coding and Information Theory, Springer, New York (1992).

[16] C.E. Shannon, A Mathematical Theory of Communication, The Bell System Technical Journal 2, (1950).

[17] A.T. da Silva, Códigos cíclicos sobre anéis de cadeia, Tese de doutorado apresentada ao Instituto de Matemática e Estatística da USP, São Paulo, (2012).

[18] R.R.M. Silva Unidades em $\mathbb{Z} C_{2 p}$ e Aplicações, Tese de doutorado apresentada ao Instituto de Matemática e Estatística da USP, são Paulo, (2012)

[19] G. Vega, Determining the Number of One-Weight Cyclic Codes when Length and Dimension are Given, Lecture Notes in Computer Science 4547, 284-293, (2007).

[20] E. Witt. Die algebraische Struktur des Gruppenringes eine endlichen Gruppe über eine Zahlenkörper, J. Für Math. 190: 231 - 245, (1952). 